\title{
Empirical Observations of Multi-modal Network-level Models: Insights from the pNEUMA Experiment
}

\author{
Mahendra Paipuri ${ }^{\mathrm{a}}$, Emmanouil Barmpounakis $^{\mathrm{b}}$, Nikolas Geroliminis $^{\mathrm{b}}$, Ludovic Leclercq $^{\mathrm{a}, *}$ \\ ${ }^{a}$ Université Gustave Eiffel, Université de Lyon, ENTPE, LICIT, Lyon, France. \\ ${ }^{b}$ Urban Transport Systems Laboratory, École Polytechnique Fédérale de Lausanne (EPFL), Station 18, CH-1015 Lausanne, Switzerland.
}

\begin{abstract}
Multi-modal interactions at the network-level remain unexplored due to the lack of high-resolution data for all transportation modes involved. The current work investigates the effect of multi-modal interactions at space-mean network speed for each mode using the dataset from $P N E U M A$ experiment that was carried out in a congested city centre network of Athens, Greece. Explanatory variables considered are the accumulation and the stopped fraction of vehicles of each mode. Firstly, a multi-modal mean speed MFD is considered by assuming that the mean speed of each mode can be expressed in terms of accumulations of all modes. The quality of multi-modal MFD fits is compared to the uni-modal ones, where the mean speed of a given mode is assumed to be a function of the accumulation of that mode only. Secondly, the classical two-fluid model is extended to multi-modal networks. An analysis on the ergodicity assumption in the context of stopped fraction is also presented. This work also introduces a network-level dynamic model that uses the stopped fraction of vehicles. This so-called extended trip-based model simulates the stop-and-go pattern of the vehicles thereby reproducing the evolution of network congestion. This work is the first to explore in this direction. The results from the classical trip-based and the extended trip-based models are compared and validated with the empirical data.
\end{abstract}

Keywords:

Multi-modal, MFD, Empirical Data, Two-Fluid Model, Ergodicity, Trip-Based Model

\section{Introduction}

The present work targets to better explain the interactions between different modes in multi-modal urban environments using massive data of vehicle trajectories from aerial footage in a congested urban network. This unique collection of empirical data allows revisiting and redeveloping network-level models based on measurable variables than theoretical conjectures that might not hold in reality. The interconnection between local interactions and their scaling to network effects will enable to develop a new era of empirical urban multi-modal traffic models. While network-level models have been studied since the 1960's and the seminal papers of Godfrey (1969) and Herman and Prigogine (1979), there have been rapid advances in the network-level modeling once dynamic Macroscopic or Network Fundamental Diagram (MFD or NFD or NMFD) models were investigated with real data for the city of Yokohama (Geroliminis and Daganzo, 2008). The MFD relates mean density to mean flow in the urban network (Mahmassani et al., 1984; Daganzo, 2007). It has evolved as a promising tool for urban management, where applications like traffic state estimation (Yildirimoglu and Geroliminis, 2014; Kavianipour et al.,

* Corresponding author

Email addresses: mahendra.paipuri@univ-eiffel.fr (Mahendra Paipuri), manos.barmpounakis@epfl.ch (Emmanouil Barmpounakis), nikolas.geroliminis@epfl.ch (Nikolas Geroliminis), ludovic.leclercq@univ-eiffel.fr (Ludovic Leclercq) 
2019; Mariotte et al., 2020), perimeter control (Ampountolas et al., 2017; Sirmatel and Geroliminis, 2018; Mohajerpoor et al., 2020; Haddad and Mirkin, 2020), congestion pricing (Zheng et al., 2016; Gu et al., 2018; Zheng and Geroliminis, 2020), route guidance (Yildirimoglu et al., 2015; Sirmatel and Geroliminis, 2017), ridesharing (Wei et al., 2020), demand management (Yildirimoglu and Ramezani, 2020) and cruising for parking (Cao and Menendez, 2015; Leclercq et al., 2017; Gu et al., 2020), etc. have been proposed based on the MFD approach.

Most of the works proposed in the literature based on the MFD assume uni-modal networks. However, urban networks are multi-modal, where different modes interact with each other in different ways. For instance, Boyaci and Geroliminis (2011); Chiabaut et al. (2014); Loder et al. (2017) showed that buses and cars have a different effect on the network dynamics in multi-modal urban networks. Similar findings (Barmpounakis et al., 2016) had been reported for Powered Two-Wheelers (PTWs) due to their smaller size and better manoeuvrability compared to the other modes. Gashaw et al. (2018) proposed microscopic traffic mixed flow model considering cars and PTWs and estimated the changes in the flow of cars for different densities of PTWs. The effect of PTWs on traffic cannot yet be fully quantified and therefore, the in-homogeneity they create may conceal a significant cause of congestion. Paipuri and Leclercq (2020) showed that it is indeed essential to differentiate the modes in the MFD-based frameworks to have accurate traffic state prediction.

Most of the works (Buisson and Ladier, 2009; Saberi and Mahmassani, 2013; Tsubota et al., 2014; Ambühl et al., 2018) focusing on the estimation of MFD using empirical empirical observations assume an uni-modal MFD Geroliminis et al. (2014) estimated the so-called bi-modal or 3D-MFD that relates the accumulation of private cars, public transport buses to the mean flow in the network based on micro-simulations. Later, Loder et al. (2017); Huang et al. (2019) showed the empirical 3DMFDs for Zurich, Switzerland and Shenzhen, China, respectively. In the stated works, the authors used either Loop Detector Data (LDD) or GPS data of private taxis in conjunction with Automatic Vehicle Locator (AVL) data of public transport vehicles to estimate bi-modal MFDs. Extending this type of bi-modal MFD to multi-modal MFD using existing techniques and data sources is not trivial. Identifying the level of interaction of local disturbances and the subsequent network effects (for example, when a bus stops for passengers) requires high-resolution data that is not readily available. Thus, the data collection challenges limited the multi-modal works to private cars and public transport buses in the literature. Recently, Paipuri et al. (2020) proposed a methodology to estimate the MFD based on mobile phone data. This framework can be used along with the mode detection techniques presented on a similar kind of reliable data in Yang et al. (2020) to estimate a multi-modal MFD in such networks. The main limitation of data from these types of sources is that they do not cover entire traffic and hence, they are available at low to moderate penetration rates. Additionally, issues related to privacy or over representation of specific modes could arise. More recently, Fu et al. (2020) estimated an empirical bi-modal MFD combining data from multiple sources for private cars, taxis and public transport buses for the city network of Shenzhen, China. Thus, there have not been any works that estimate a true multi-modal mean speed MFD by considering all urban network modes.

A novel way of traffic data collection in the recent past is using Unmanned Aerial Systems (UAS) or drones (Barmpounakis et al., 2016). Several previous works (Barmpounakis et al., 2018; Khan et al., 2018; Barmpounakis et al., 2019) used a single drone to monitor the urban traffic at the intersections or specific arterials. Recently, Barmpounakis and Geroliminis (2020) extended this idea to monitor the traffic at the network scale using a swarm of drones in Athens, Greece. This experiment, named pNEUMA (New Era of Urban traffic Monitoring with Aerial footage), provides a unique set of data, where more than 100000 trajectories, with their mode identified, in a large urban area with more than 200 links and 60 bus stops were monitored. The main advantage of this data compared to probe vehicles or smartphones is the complete network coverage 
and the quality of the individual trajectories, where almost every vehicle is tracked. Thus, it can be used in both microscopic and macroscopic studies of traffic flow problems like calibrating lane changing models, determining the impact of location of loop detectors on the estimation of MFD, etc.

In the current work, this dataset is used to investigate the multi-modal interactions of all modes with respect to their mean speeds. The dynamic influence of multi-modal interactions in network space-mean speed is investigated using two sets of explanatory variables, namely accumulations as per the MFD theory and network-wide stopped fractions of vehicles as proposed in the two-fluid model by (Herman and Prigogine, 1979). Most of the works proposed based on the two-fluid model in the literature (Jones and Farhat, 2004; Vo et al., 2007; Dixit, 2013) considered only private cars in the network. Chakraborty and Srinivasan (2016) used the GPS data of public transport buses to study the impact of measurement errors on the two-fluid model mean speed curves. Besides, there had been very few works (Herman and Ardekani, 1984; Ardekani and Herman, 1987) validating the ergodic assumption used in the two-fluid model. The present dataset can shed some insights on the ergodicity assumption as trajectories of all types of vehicles are known a priori. Thus, the current work also addresses this research gap by presenting a study on ergodicity assumption in estimating the stopped fraction of vehicles. Besides, the influence of penetration rates on the estimation of stopped fractions is also analysed.

Multi-modal mean speed regression fits are essential to understand the interactions between different modes at the network level. These are critical inputs to the macroscopic dynamic models used to predict the future traffic states and apply different control actions. While mean speed vs. accumulation fits can be directly used in the context of the MFD-based models, no attempts have been made to use mean speed prediction methods based on the two-fluid model, i.e., using mean speed vs. network-wide stopped fraction fit as an input to the macroscopic model. Thus, this work is the first step to explore in this research direction by proposing the multi-modal two-fluid model and comparing it with the multi-modal MFD model. It is worth noting that this is possible due to the unprecedented coverage of current dataset that have penetration rates higher than $99 \%$. In the context of the current study, the trip-based MFD model is extended to segregate the two typical vehicular states during their trips (running and stopped) and the modelling and application of the single-mode and bi-modal MFDs are extended to a general multi-modal mean speed vs. accumulation of each individual mode. Then, building on the two-fluid model concept, a multi-modal two-fluid model is developed, where the speed of each mode depends on the stopped fraction of vehicles of all the other modes. Finally, an extended trip-based model that replicates the stop-and-go pattern of the vehicles is proposed.

The paper is organized as follows: Section 2 discusses the details about the network and the data, Section 3 compares the mean speeds and mean running speeds for different modes and presents the regression analysis of mean speeds using accumulations as explanatory variables, Section 4 provides a detailed discussion on the regression analysis of mean speeds using stopped fractions as explanatory variables, Section 5 proposes the extended trip-based model and validates it using the empirical data and finally, Section 6 brief conclusions of the work.

\section{Data Description and Processing}

Figure 1a shows the region under consideration in the down town area of Athens city. Fig. 1b presents the link-level description of down town Athens, where links highlighted in blue falls under the monitored region. A swarm of 10 drones was used to monitor the central business district of Athens that covers an area of $1.4 \mathrm{~km}^{2}$. The total length of the road network in the considered area is around $78 \mathrm{~km}$ and there are a total of 63 bus stops. The experiment was carried out on five weekdays, 


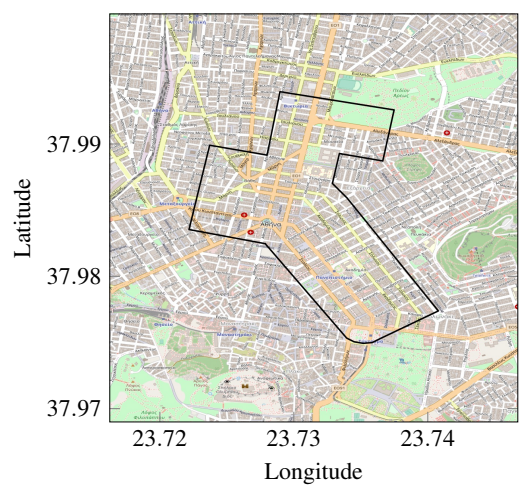

(a)

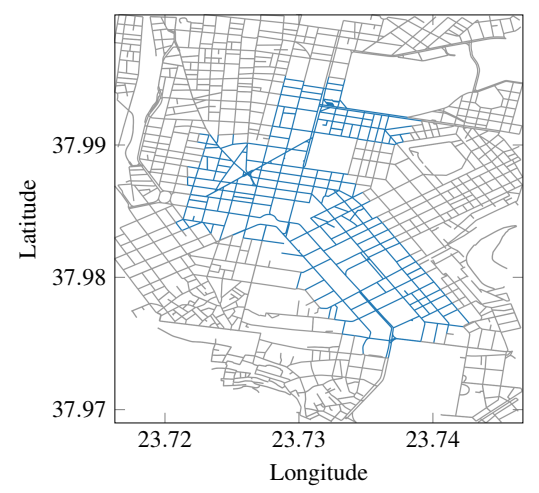

(b)

Figure 1: Athens network: map of the area and its link level description. (a) Map of Athens, Greece @OpenStreetMap 2020. (b) Link level representation of Athens. Links in blue are present inside the considered area.

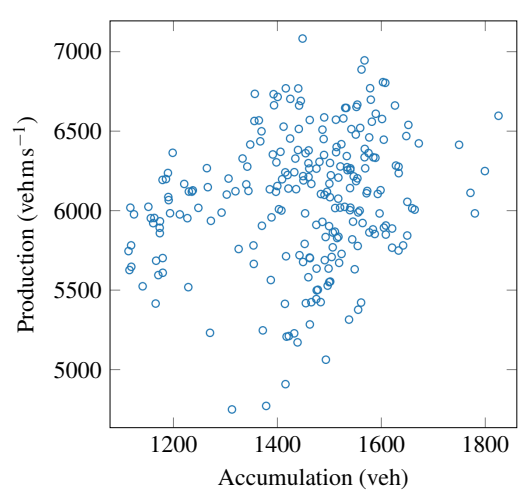

(a)

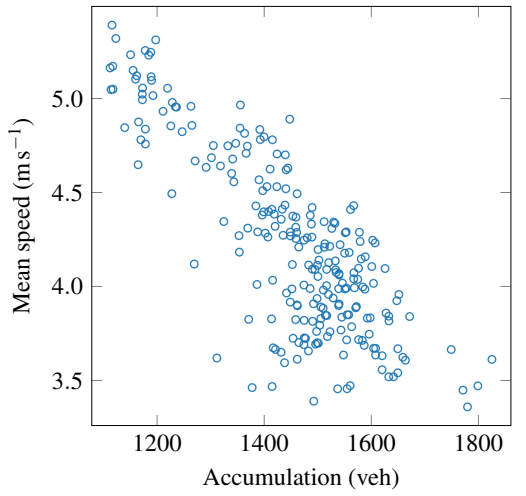

(b)

Figure 2: 2D MFDs of considered region. (a) Production MFD. (b) Mean speed MFD.

namely October 19, 2018, October 24, 2018, October 29, 2018, October 30, 2018 and November 1, 2018. In the present work, only data from the last four days of the experiment is used. On the first day, the morning peak hour from 08:30 AM to 11:00 AM was monitored, whereas on the remaining three days, the traffic stream from 08:00 AM to 10:30 AM was monitored.

The video recordings were processed to extract the individual trajectories of each vehicle along with its mode. In the current dataset, six different modes are identified: private cars, taxis, buses, medium vehicles, heavy vehicles, and PTWs. Although the raw data has a higher sampling frequency of $0.04 \mathrm{~s}$, for the current study, it is eventually downgraded to $1 \mathrm{~s}$ in order to ease the data processing. More details about the experimental setup can be found in Barmpounakis and Geroliminis (2020) and hence, they are omitted here.

Since the drones have a limited battery life, the recordings were intermittent. Drones were brought back to change the batteries and thus, $12 \mathrm{~min}$ to $15 \mathrm{~min}$ of recording was lost inside each $30 \mathrm{~min}$ period. Hence, for every $30 \mathrm{~min}$ period the active recording time is only around $15 \mathrm{~min}$ to $17 \mathrm{~min}$. It is noticed that not all drones were recording either at the beginning or towards the end of active recording periods due to synchronization issues. Only data corresponding to complete drone coverage is considered to minimize the bias in estimating macroscopic variables in the current work. According to the 
generalized definitions of Edie (1963); Saberi et al. (2014), average flow $(q)$ and average density $(k)$ are defined as,

$$
q=\frac{\sum_{i=1}^{M} T D_{i}}{L_{n} \Delta T} \text { and } k=\frac{\sum_{i=1}^{M} T T_{i}}{L_{n} \Delta T},
$$

where $T D_{i}$ and $T T_{i}$ are travelled distance and travelled time of vehicle $i$ during a given aggregation interval $\Delta T, L_{n}$ is the total length of the network and $M$ is the total number of trajectories. In the present work, an aggregation interval of $1 \mathrm{~min}$ is assumed. The mean speed of the network can be estimated as the ratio of average flow to average density. The computed $k$ and $q$ are used to estimate vehicle accumulation $\left(L_{n} k\right)$ and production $\left(L_{n} q\right)$, respectively. Vehicle accumulation can be defined as the total number of vehicles present in the network in a given aggregation interval, whereas production is average distance travelled by all vehicles in the network during the aggregation interval. Fig. 2 presents the production and mean speed MFDs considering all vehicles irrespective of modes in the considered region. Fig. 2a shows that most of the data points are in the network saturation regime of the production MFD. The active drone recordings start either at 08:00 AM or 08:30 AM, where the network is expected to be saturated. Thus, the data points corresponding to the free-flow regime are not obtained in the experiment. The mean speed MFD shown in Fig. $2 \mathrm{~b}$ has a mean speed range of $1.5 \mathrm{~ms}^{-1}$ at the network saturation. Similarly, production MFD shows a scatter of $2000 \mathrm{veh} \mathrm{m} \mathrm{sec}^{-1}$ at the peak hour. It is worth noting that no smoothening techniques were used to present the results and thus, compared to previous works in the literature, relatively low scatter MFDs are obtained for both production and mean speeds.

It is also noticed that certain trajectories, especially of cars and taxis, have very low mean speeds, less than $2 \mathrm{~ms}^{-1}$, over their entire trajectory. Given that $1 \mathrm{~m} \mathrm{sec}^{-1}$ to $1.5 \mathrm{~m} \mathrm{sec}^{-1}$ is a nominal walking speed, such a low mean speed over the whole trip is rather unusual. This is due to the vehicle tracking algorithm used to extract the trajectories from drone videos. The vehicle tracking algorithm does not stop tracking a vehicle even after it has stopped. For instance, consider a vehicle that was moving for the first 30 seconds of the recording and then was parked for the rest 14 minutes. The trajectory data will contain the whole $14 \mathrm{~min}$ and $30 \mathrm{sec}$. In the case of taxis, this might happen when they are stopped in a taxi stop, move for 4 meters to occupy the next available slot and then stop again until a new customer/passenger arrives. At the same time, these very low mean speeds are not due to long queues in the spillbacks that can typically occur during peak hours. Spillbacks and long queues occur in the network under consideration, but there is still heterogeneity and the network is really far from gridlock conditions. Thus, long queues might propagate for a few intersections, but do not cover the whole network. This can be verified by space-time diagrams presented in Barmpounakis and Geroliminis (2020, Figs. 12, 13 and 18), which were plotted for the same dataset. Thus, these set of trips are flagged for further investigation and data cleaning. The following technique is adopted for the processing these low-speed trajectories: Firstly, all the trajectories that have mean speed of less than $2 \mathrm{~m} \mathrm{sec}^{-1}$ over the entire trajectory are flagged for further scrutiny. In the current case, these set of trips are around 8 to $9 \%$. The inherent assumption is that the vehicle made a stop for a significant time and hence, a very low mean speed over the entire trip is observed. The global idea is to identify the time instances corresponding to these stoppages and remove them from the dataset. In this set of flagged trajectories, vehicles that remain stationary (zero instantaneous speed) for more than $3 \mathrm{~min}$ are further filtered out. Most of the signals in the area of investigation have timing cycles between 1 min to $2 \mathrm{~min}$ and thus, a threshold of $3 \mathrm{~min}$ is chosen in the current case. If these stoppages occur at the beginning or at the end of the trips, trajectories are simply truncated to remove the data points corresponding to these timestamps. On the other hand, if they occur in the middle of the trip, the trajectory is divided into two different ones by neglecting the timestamps of stoppage time. Thus, this process is done only for the trips with very low mean speed over their entire trajectories. Indeed, a sensitivity analysis 
is done with different threshold times. It is observed that using longer thresholds increases the scatter in the MFD as they introduce artificial bias to the mean speed estimation. Using a threshold less than the signal timing settings would filter out the traffic-related stops.

\section{Uni-modal vs. Multi-modal Mean Speed MFDs}

In this section the importance of multi-modal MFD in understanding and evaluating the multi-modal performance of the urban system is demonstrated. Multi-modal MFD can be used to determine if the underlying networks is in a state that produces the desired accessibility levels for all modes of transport. Towards this direction mean speed vs. accumulation relation for each individual mode is investigated to show if there is a clear trend of congestion.

Figure 2 shows that the current dataset covers only a fraction of the production-accumulation plane. The experiment monitors only at the morning peak hour and data from the off-peak hours are missing. In order to determine robust multi-modal mean speed fit, it is necessary to have the data that cover a wide range of accumulations for each mode pair. However, this requires a longer duration of the experiment, which was not possible during the first execution and in reality, accumulations of each mode tend to operate within certain regions. In order to compute the mean speed of a given mode $j$, generalized definitions of Edie (1963) presented in eq (1) are modified accordingly to include only the trajectories of vehicles that belong to that mode to estimate average flow $\left(q_{j}\right)$ and average density $\left(k_{j}\right)$ of that mode.

Figure 3 presents the mean speed $\left(v_{j}\right)$ of a given mode as a function of the accumulation $\left(n_{j}\right)$ of that mode $j$. The subscripts $j \in\{c, t, b, m, h, p\}$ represent cars, taxis, buses, medium vehicles, heavy vehicles and PTWs, respectively. Firstly, it can be noticed that cars are a significant fraction of the vehicles in the network, followed by taxis and PTWs. Taxis and PTWs have minimal scatter in the MFD compared to other modes. On the contrary, heavy vehicles show significant scatter in the MFDs. In the case of heavy vehicles, the range of accumulation is very low, approximately $30 \mathrm{veh}$, which might contribute to the scatter in the MFD. Since there are very few heavy vehicles in the network compared to other modes, they are neglected in the current analysis. Several factors influence the scatter in the bus MFD. Buses make more frequent stops than other modes to aboard and alight the passengers at the bus stops. Simultaneously, if there are dedicated bus lanes, they can travel faster than their counterparts during the congested periods. Thus, the combination of these two effects results in a broader range of speeds and, consequently, scatter in the mean speed MFD. Finally, cars, taxis and PTWs show a satisfactory trend with an acceptable scatter in the MFDs.

Figure 3 shows that PTWs travel at higher speeds amongst the modes considered and heavy vehicles have the lowest. However, this direct comparison is biased as buses and taxis have service-related stops in addition to the stops at signalized intersections and congested links. In order to compare the "true" mean speed of each mode, the so-called mean running speed $\left(v_{r, j}\right)$ of each mode $j$ is estimated. The mean running speed can be defined as the average speed of all moving vehicles of a given mode in a given aggregation interval. In other words, the mean running speed is computed by excluding all the stopped vehicles during their trajectories. It is to be noted that the accumulations shown in the plots correspond to total number of vehicles including both running and stopped. In Fig. 3, the data points in orange show the mean running speed MFD of each mode. Among all the different modes considered, it is noticed that buses show a bigger difference between mean speed and mean running speed. This can be due to the service-related stops that are inherent for buses. At the same time, PTWs also show a difference comparable to buses owing to their faster speeds during movements compared to other modes. As there are only few heavy vehicles during the monitored peak hours and they are not making service-related stops, they are excluded in 


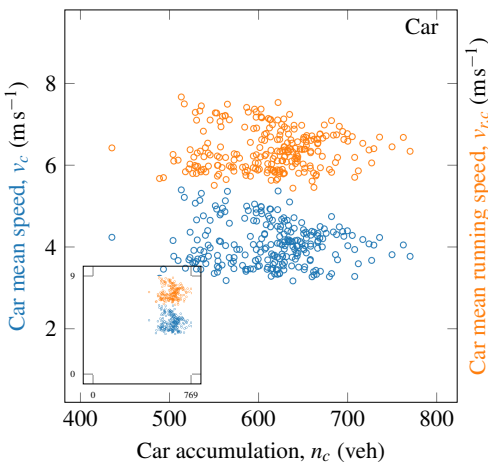

(a)

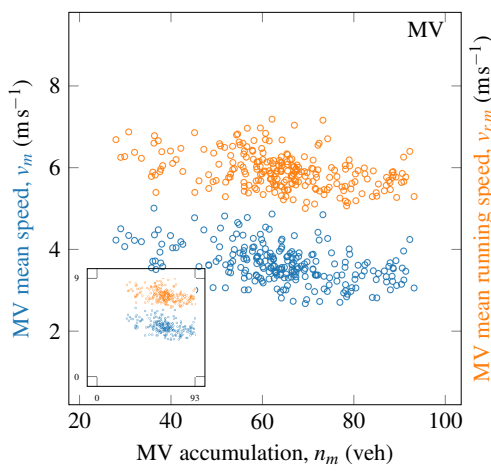

(d)

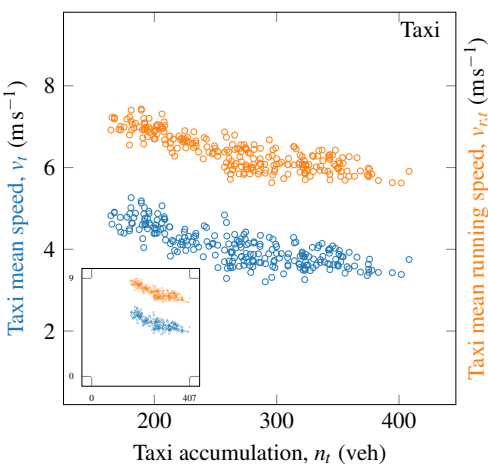

(b)

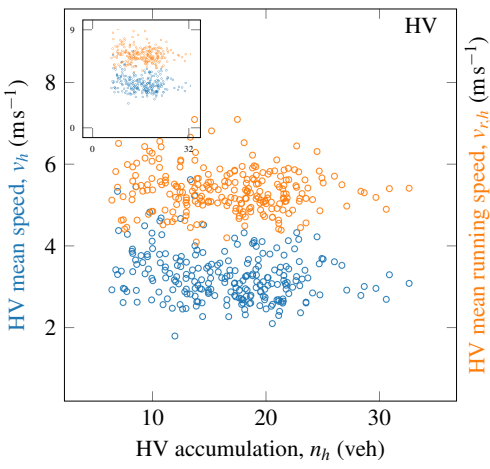

(e)

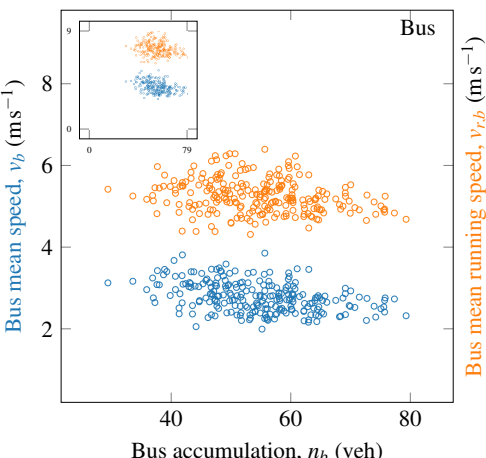

(c)

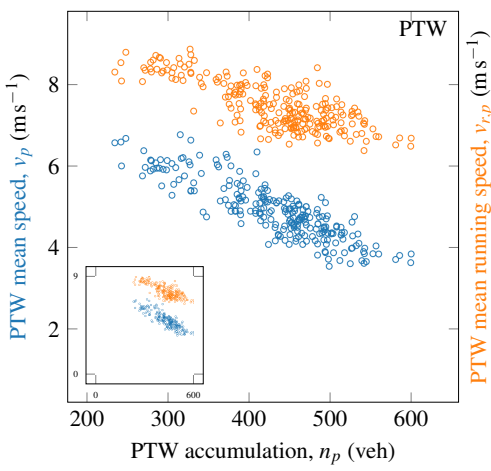

(f)

Figure 3: Partial mean speed $\left(v_{j}\right)$ and mean running speed $\left(v_{r, j}\right)$ MFDs for mode $j$. (a) Car. (b) Taxi. (c) Bus. (d) Medium Vehicle (MV). (e) Heavy Vehicle (HV). (f) PTW.

the current analysis due to the sample size. Appendix A shows the comparison between the mean speed and mean running speed of each mode is compared to that of cars to deduce the network's traffic characteristics.

In the previous works that estimated the bi-modal MFDs (Geroliminis et al., 2014; Loder et al., 2017), mean speed of entire network is expressed as a function of accumulations of private cars and public transport vehicles. However, it was shown in Paipuri and Leclercq (2020) that segregating mean speed of each mode is essential for accurate traffic state estimation in the MFD-based models. Thus, in the current work mean speed of each mode is assumed to be a function of accumulation of all modes in the network. Although very detailed data is available in the present case, the coverage in terms of traffic states remains limited. Hence, a linear functional form (Ampountolas et al., 2017; Loder et al., 2017) is chosen and mean speed of each mode is expressed as linear combination of all mode in the current work. If the data across the wider ranges of accumulation is available, it is necessary to consider a piece-wise linear functional form to approximate the mean speed accurately. The linear functional form assumed in the present work can be expressed as follow,

$$
v_{j}=v_{f, j}+\alpha_{c, j} n_{c}+\alpha_{t, j} n_{t}+\alpha_{b, j} n_{b}+\alpha_{m, j} n_{m}+\alpha_{p, j} n_{p}, \quad \forall j=\{c, t, b, m, p\}
$$

where $v_{j}$ is mean speed of mode $j, v_{f, j}$ is free-flow speed of mode $j$. The regression coefficient $\alpha_{k, j}$ represents the marginal negative effect of mode $k$ on the mean speed of mode $j$. Thus, this functional form has a clear physical meaning and it is possible to quantify the relative importance of all modes on the mean speed of each mode. Besides, uni-modal approach is also considered where the mean speed of a given mode is assumed to depend on that mode only, i.e., regression coefficients 
in (2) can be expressed as,

$$
\alpha_{k, j}= \begin{cases}1 & \text { if } k=j, \\ 0 & \text { else. }\end{cases}
$$

The mean speed fits from both approaches above are compared to demonstrate the importance of considering the multi-modal interactions.

All regression coefficients in (2) must be negative to respect the physics of the traffic (Geroliminis et al., 2014). Thus, in order to determine the fit coefficients $\alpha_{k, j}$, Non-Negative Least Squares (NNLS) (Lawson and Hanson, 1995) is used in the present work. This method is a variant of constrained linear least squares, where a lower bound of zero is applied to the regression coefficients. It is straightforward to use this method to obtain negative regression coefficients in the current context. Least-squares methods tend to fail when there is a large set of explanatory variables and highly correlated. Partial Least Squares (PLS) (Geladi and Kowalski, 1986; Wold, 1992) technique addresses this limitation of multicollinearity between the explanatory variables. The idea behind the PLS method is to decompose the independent and dependent variable matrices using Principal Component Analysis (PCA) and then perform the regression between the projected components. It is noticed that the regression coefficients obtained by NNLS and PLS methods are very similar and hence, only results of the NNLS method are presented to avoid redundancy. At the same time, it is concluded that the multicollinearity between explanatory variables, i.e., accumulations of different modes is weak in the current dataset.

Figure. 4 shows the coefficients of mean speed linear regression fits for different modes using NNLS method. The accumulation of each mode is standardized using the mean and standard deviation of that mode. This permits to compare the impact of each mode on the mean speed of a given mode. The Root Mean Squared Relative Error (RMSRE) is defined as,

$$
\operatorname{RMSRE}=\sqrt{\frac{1}{w} \sum_{l=1}^{w}\left(\frac{\hat{v}_{l}-v_{l}}{v_{l}}\right)^{2}},
$$

where $\hat{v}_{l}$ and $v_{l}$ are the $l^{\text {th }}$ predicted and actual observations, respectively and $w$ is total number of observations. Firstly, it is clear from the plots that all the coefficients are negative as expected. It is also clear that PTWs have the highest and buses have the lowest free-flow speeds amongst the modes. As discussed earlier, the mean speeds of cars and taxis are very similar and that conclusion is reiterated here, where free-flow speeds of both modes are very similar. The values of $R^{2}$ and RMSRE suggest that the mean speeds of cars, taxis and PTWs resulted in relatively tight and accurate fits. In the case of medium vehicles and buses, low $R^{2}$ values are obtained. This is due to the relatively small range of accumulations noticed in these two modes. The dwelling buses in the bus stops can introduce a significant scatter in the mean speed estimation too. A new explanatory variable in the form of total dwell time is added to the regression analysis of buses to account for the dwell times of the buses in each aggregation interval. However, it is noticed that neither the $R^{2}$ nor the RMSRE improves by adding the dwell time to the explanatory variables and hence, the results are omitted here. Another reason for larger errors in bus speed prediction is the fact that the number of buses circulating is smaller (accumulation ranges between 40 and 80 buses) and they are not moving in all parts of the network. A more detailed analysis could shed more light by considering only the roads that buses are moving. This will be a future research direction, as it will require significant effort to associate trajectories of vehicles with links. On the other hand, RMSRE values for all modes fall within 6 to $12 \%$, which is reasonable.

It can be noticed that taxis have a significant negative impact on all other modes except PTWs. This can be due to the aggressive driving behavior of taxi drivers compared to buses and MVs. Similarly, PTWs significantly impact the mean speed 


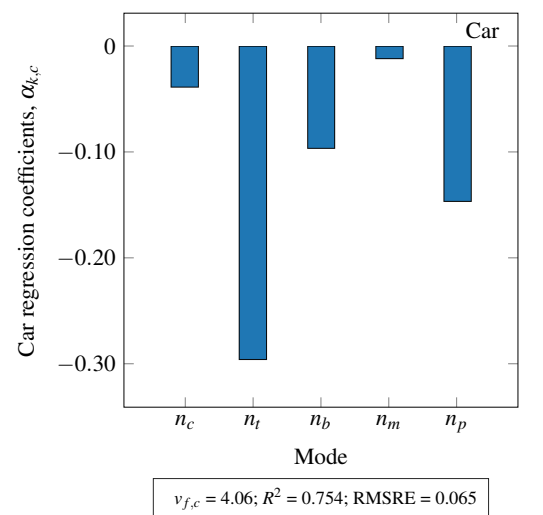

(a)

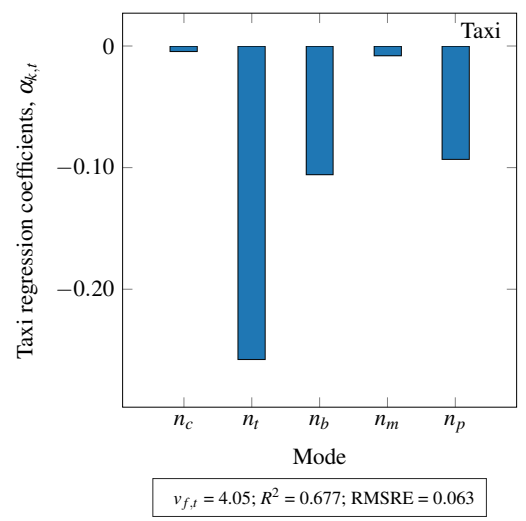

(b)

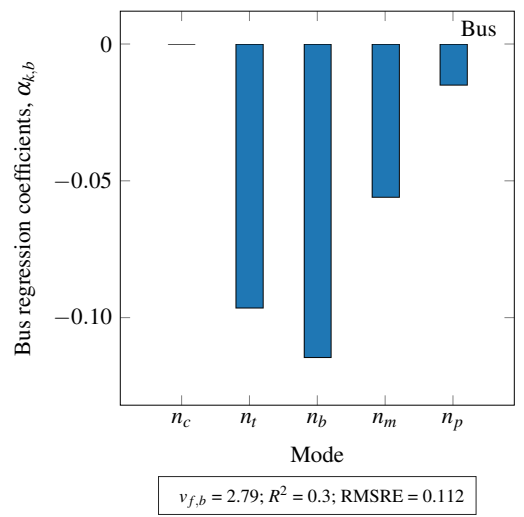

(c)

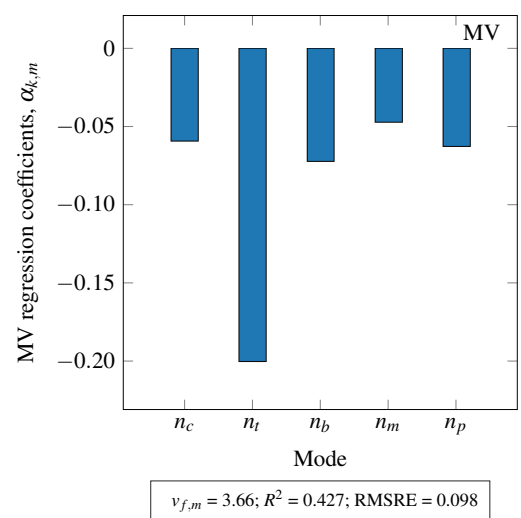

(d)

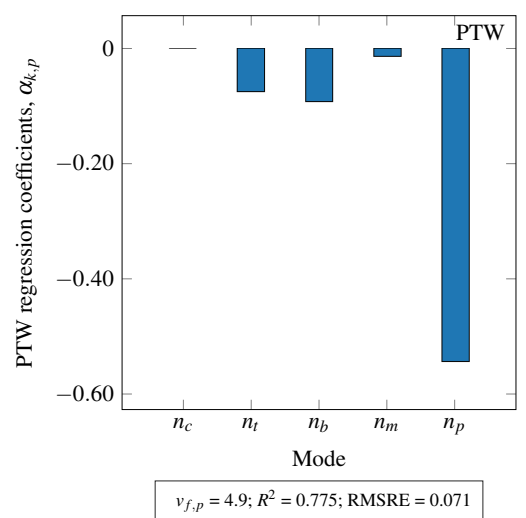

(e)

Figure 4: Coefficients of linear regression fits for mean speed using NNLS for each mode. Free flow speed $v_{f, j}$ is in $\mathrm{ms}^{-1}$. (a) Car. (b) Taxi. (c) Bus. (d) Medium Vehicle (MV). (e) PTW. 
Table 1: Statistical p-values, mean speed in $\mathrm{ms}^{-1}, R^{2}$ and RMSRE values of uni- and multi-modal mean speed fits for all modes using NNLS method.

\begin{tabular}{|c|c|c|c|c|c|c|c|c|c|c|c|c|}
\hline \multirow{3}{*}{ Mode } & \multicolumn{6}{|c|}{ p-values } & \multicolumn{2}{|c|}{$v_{f, j}$} & \multicolumn{2}{|c|}{$R^{2}$} & \multicolumn{2}{|c|}{ RMSRE } \\
\hline & \multirow{2}{*}{$\frac{\text { Uni- }}{n_{m}}$} & \multicolumn{5}{|c|}{ Multi- } & \multirow{2}{*}{ Uni- } & \multirow{2}{*}{ Multi- } & \multirow{2}{*}{ Uni- } & \multirow{2}{*}{ Multi- } & \multirow{2}{*}{ Uni- } & \multirow{2}{*}{ Multi- } \\
\hline & & $n_{c}$ & $n_{t}$ & $n_{b}$ & $n_{m}$ & $n_{p}$ & & & & & & \\
\hline Car & 0.01 & 0.01 & 0 & 0 & 0.60 & 0 & 4.06 & 4.06 & 0.01 & 0.75 & 0.125 & 0.065 \\
\hline Taxi & 0 & 0.80 & 0 & 0 & 0.72 & 0 & 4.05 & 4.05 & 0.60 & 0.68 & 0.071 & 0.063 \\
\hline Bus & 0 & 0.14 & 0 & 0 & 0.06 & 0.48 & 2.79 & 2.79 & 0.15 & 0.30 & 0.123 & 0.112 \\
\hline MV & 0 & 0.01 & 0 & 0 & 0.12 & 0.07 & 3.66 & 3.66 & 0.19 & 0.43 & 0.114 & 0.098 \\
\hline PTW & 0 & 0 & 0.06 & 0 & 0.63 & 0 & 4.90 & 4.90 & 0.04 & 0.78 & 0.074 & 0.071 \\
\hline
\end{tabular}

of cars, taxis and medium vehicles. This is again due to the filtering behavior of the PTWs, which negatively impact the speeds of other modes. It is also inferred that the mean speed of PTWs is majorly impacted by other PTWs in the network, as they can form platoons to move faster compared to other vehicles.

To show the importance of a multi-modal approach, the RMSRE errors of the multi-modal mean speed fits are compared with the uni-modal ones estimated using eqs. (2) and (3). Table 1 presents the $R^{2}$ and RMSRE for uni- and multi-modal mean speed fits using NNLS technique. Firstly, it can be noticed that there is an improvement in both $R^{2}$ and RMSRE values going from uni-modal to multi-modal approach. Notably, in the case of cars, there is a significant improvement in $R^{2}$ and RMSRE values using multi-modal fit, where the error is almost halved compared to the uni-modal one. Following, taxis and medium vehicles show a 10\% - 15\% decrease in the RMSRE when the multi-modal functional form is assumed. It is observed that the buses and PTWs show minor improvement with the current dataset. However, $R^{2}$ value of the buses doubled in the case of multi-modal fit, although the improvement in RMSRE is marginal. In the case of PTWs, it is clear from Fig. 4e that the other PTWs majorly influence their mean speed as they tend to form platoons and the interactions from other modes is rather minimal. Thus, there is no significant improvement resorting from the uni-modal to multi-modal approach for PTWs. Finally, it can be concluded that the multi-modal approach gives better mean speed estimation compared to the uni-modal one, even though the improvement is not very significant for some modes.

Finally, it can be concluded that multi-modal mean speed MFD describes the variation of mean speed over the uni-modal counterpart, where the improvement is quite pronounced for some modes. However, it is worth noting that data coverage is the main limitation of the current analysis. Nevertheless, the current regression analysis gives satisfactory results. However, there is room for improvement in predicting the mean speed of some modes, mainly buses and medium vehicles. Thus, in the following section, another functional form using stopped fractions instead of accumulations as explanatory variables is investigated.

\section{A Multi-modal Two-fluid Model}

\subsection{Background}

A functional form between mean speed and accumulations is not only way to characterize the traffic behaviour at networklevel and there are other expressions that have been proposed in the literature. In particular, the two-fluid theory proposed by Herman and Prigogine (1979) for the town traffic is based on the kinetic theory of multi-lane highway traffic (Prigogine 
and Herman, 1971). The traffic in a city network can be assumed to have two parts: running vehicles and stopped vehicles due to congestion, traffic signals, stop signs, etc., but not parked vehicles. Two parameters characterize the two-fluid model, namely minimum travel time $\left(\frac{1}{v_{f, r}}\right)$ and an indicator of the quality of traffic in the network $(\check{n})$. The model is based on two assumptions:

1. The mean running speed $\left(v_{r}\right)$ of vehicles in the network is proportional to the fraction of vehicles $\left(f_{r}\right)$ that are moving.

2. The fraction of the time stopped of a given vehicle is equal to the average fraction of the homogeneous population of vehicles $\left(f_{s}\right)$ over the same period. It is clear that $f_{r}+f_{s}=1$.

The first assumption can be expressed mathematically as follows,

$$
v_{r}=v_{f, r}\left(f_{r}\right)^{\check{n}} \equiv v_{f, r}\left(1-f_{s}\right)^{\check{n}}
$$

255

where $v_{f, r}$ is the free-flow running speed. As the mean speed can be defined as $v=v_{r} f_{r}$, eq. (5) can be re-written as,

$$
v=v_{f, r}\left(f_{r}\right)^{\check{n}+1} \equiv v_{f, r}\left(1-f_{s}\right)^{\check{n}+1} .
$$

The essential difference compared to the regression analysis presented in Section 3 is that the explanatory variable for the mean speed is no longer the vehicle accumulations but the fraction of stopped (or running vehicles). However, this variable is difficult to monitor in reality and thus, it is necessary to estimate this fraction from other variables that are easier to measure in the field, i.e., the accumulations.

The second assumption leads to the following expression,

$$
f_{s}=\frac{T_{s}}{T}
$$

where $T_{S}$ is the mean stopped time per unit distance and $T$ is the trip time per unit distance. Substituting eq. (7) in eq. (6) and using relations $v=\frac{1}{T}, T_{f}=\frac{1}{v_{f, r}}$ and $T=T_{f}+T_{s}$, the following expressions can be deduced:

$$
T_{r}=T_{f}^{\frac{1}{\tilde{n}+1}} T^{\frac{\check{n}}{\tilde{n}+1}} \text { and } T_{s}=T-T_{f}^{\frac{1}{\tilde{n}+1}} T^{\frac{\check{n}}{\tilde{n}+1}} \text {. }
$$

It is to be noted that the variables in the two-fluid model are always meant to be mean values taken over the entire network. Eq. (8) represents conventional two-fluid model. A higher value of $\breve{n}$ for a given value of $T_{f}$ leads to lower mean running speeds and thus, more congested traffic. Several field studies showed that the value of $\breve{n}$ varies between 0.8 to 3 , where smaller $\check{n}$ indicates better operating conditions for traffic flow (Williams, 2001).

As a first step, the two-fluid model is applied to the current dataset per mode to investigate if the assumptions of the two-fluid model hold on the modal data. This is referred to as uni-modal two-fluid regression. Simultaneously, the classical two-fluid model is also calibrated by treating all modes alike to estimate the two-fluid parameters for the entire network under consideration. The so-called uni-modal and classical two-fluid regressions are then compared to investigate if segregating the modes can improve the estimation of model parameters.

\subsection{Multi-modal two-fluid model}

Most of the works based on the two-fluid model were proposed at the network level without considering the multimodality. Herman and Ardekani (1984) presented the results of a controlled experiment involving buses and cars in the 
context of the two-fluid model. It was reported that buses have a considerably higher value of $\breve{n}$ compared to cars. The authors claimed that the differences in the two-fluid parameters result from additional acceleration-deceleration cycles of the buses during the dwelling. However, they applied the two-fluid model to cars and buses independently and did not account for their interactions. For instance, in eq. (6), it is a very crude approximation to assume that the mean speed of a given mode depends only on the stopped fraction of vehicles of that mode itself, especially in multi-modal urban networks. At the same time, the two-fluid model does not explain the scatter in the city traffic data presented in Ardekani (1984).

The first assumption of the original two-fluid model is modified as: "The mean running speed $\left(v_{r, j}\right)$ of vehicles of mode $j$ is proportional to the fractional of vehicles of all modes that are moving". This modified assumption is the basis of the multi-modal two-fluid regression. Mathematically, it can be expressed as,

$$
v_{r, j}=v_{f, r, j} \prod_{k \in \mathscr{M}}\left(1-f_{s, k}\right)^{\check{n}_{k, j}}, \forall j \in \mathscr{M} \text { and } \mathscr{M}=\{c, t, b, m, p\}
$$

where $v_{f, r, j}$ is free-flow running speed of mode $j, f_{s, k} \forall j$ is the stopped fraction of vehicles and $\breve{n}_{k, j}$ is two-fluid parameter of mode $k$. Consequently, using the relation $v_{j}=v_{r, j}\left(1-f_{s, j}\right)$, the mean speed of mode $j$ can be expressed as,

$$
v_{j}=v_{f, r, j}\left(1-f_{s, j}\right) \prod_{k \in \mathscr{M}}\left(1-f_{s, k}\right)^{\check{n}_{k, j}}, \forall j \in \mathscr{M} \text { and } \mathscr{M}=\{c, t, b, m, p\}
$$

When all the vehicles of all modes are moving, $f_{s, j}=0$ and therefore, the mean speed of that mode is equal to free-flow running mean speed $\left(v_{f, r, j}\right)$. Similarly, if all the vehicles are stopped, $v_{j}=0$ according to eq. (10). Hence, the assumption in eq. (9) satisfies the boundary conditions. The multi-modal extension differs from the original model when $f_{s, j}$ is between 0 and 1. Consider a case when $f_{s, j}=0$, i.e., all the vehicles of mode $j$ are moving, but there are stopped vehicles of other modes, i.e., $f_{s, k} \forall k \neq j$ are non-zero. In that scenario, the mean speed of mode $j$ will be lower than free-flow running speed and its magnitude depends on the stopped fraction $\left(f_{s, k}\right)$ and the corresponding $\check{n}_{k, j}$ of each mode. For a given number of total vehicles, different combinations of stopped fractions between different modes give different mean speeds as per eq. (10). On the other hand, the original two-fluid model depends only on the stopped fraction of all vehicles irrespective of the mode and always yields the same mean speed. Hence, eq. (10) can explain the scatter in the data.

Now using the relations $v_{j}=\frac{1}{T_{j}}, v_{f, r, j}=\frac{1}{T_{f, r, j}}$ and $f_{s, k}=\frac{T_{s, j}}{T_{j}}$, stopped time per unit distance can be expressed in terms of trip time per unit distance as follows,

$$
T_{s, j}=T_{j}-\frac{T_{f, r, j}^{\frac{1}{\tilde{n}_{j, j}+1}} T_{j}^{\frac{\check{n}_{j, j}}{\tilde{n}_{j, j}+1}}}{\prod_{k \in \mathscr{M}, k \neq j}\left(1-f_{s, k}\right)^{\check{n}_{k, j}+1}}, \forall j \in \mathscr{M} \text { and } \mathscr{M}=\{c, t, b, m, p\} .
$$

Here $T_{s, j}$ is a function of travel times of all modes and hence, interactions between different modes are taken into account. Notice that the two-fluid parameter $\breve{n}_{k, j}$ quantifies the influence of stopped vehicles of mode $k$ on the mean speed of mode $j$. Like the previous study, empirical data is used to estimate the model parameters in eq. (10). The non-linear relation in eq. (10) can be transformed into linear by using log-transformation and so, linear regression can be used on the resulting transformed equation.

As the trajectory data of each vehicle is available, it is possible to estimate the total number of vehicles and number of stopped vehicles within each aggregation interval. Using generalized definitions of Edie (1963), total number of vehicles $(n)$ 
Table 2: Two-fluid parameters for different modes using uni-modal and classical approaches using NNLS technique.

\begin{tabular}{c|c|c|c|c|c}
\hline Mode & $v_{f, r, j}$ in $\mathrm{ms}^{-1}$ & $\check{n}_{j}$ & $R^{2}$ & RMSRE (Uni-) & RMSRE (Classical-) \\
\hline Car & 10.890 & 1.184 & 0.880 & 0.031 & 0.045 \\
Taxi & 9.805 & 0.958 & 0.794 & 0.035 & 0.050 \\
Bus & 5.785 & 0.158 & 0.656 & 0.076 & 0.119 \\
MV & 7.849 & 0.578 & 0.769 & 0.046 & 0.081 \\
PTW & 9.152 & 0.479 & 0.802 & 0.040 & 0.080 \\
\hline Classical & 10.900 & 1.092 & - & - & - \\
\hline
\end{tabular}

and total number of stopped vehicles $\left(n_{s}\right)$ can be computed as,

$$
n=\frac{\sum_{i=1}^{M} T T_{i}}{\Delta T} \text { and } n_{s}=\frac{\sum_{i=1}^{M} S T_{i}}{\Delta T},
$$

where $S T_{i}$ is the stopped time of vehicle $i$ during a given interval. In the present work, a vehicle is considered as stopped when the instantaneous speed is less than $2 \mathrm{kmh}^{-1}$. Using eq. (12), $n$ and $n_{s}$ can be computed for each aggregation interval and consequently, stopped fraction of vehicles can be estimated as $f_{s}=\frac{n_{s}}{n}$.

\subsection{Uni-modal and multi-modal two-fluid models results}

Besides multi-modal two-fluid model, the original two-fluid model assumptions are applied to each mode separately to investigate if they hold true in the case of modal segregation. Thus, eqs. (6) and (8) can be modified to apply for each mode as follows,

$$
v_{j}=v_{f, r, j}\left(1-f_{s, j}\right)^{\check{n}_{j}+1} \text { and } T_{s, j}=T_{j}-T_{f, j}^{\frac{1}{\tilde{n}_{j}+1}} T^{\frac{\check{n}_{j}}{n_{j}+1}} \forall j=\{c, t, b, m, p\}
$$

Finally, the original two-fluid model is also calibrated by assuming all modes to be alike. As stated earlier, log-transformation can be used for the mean speed in eq. (13) to transform the non-linear equation into a linear one. Table 2 shows the estimated fit parameters for each uni-modal and the classical two-fluid model using the NNLS method. The RMSRE is estimated using 5 both uni-modal and classical two-fluid approaches. RMSRE (Uni-) in the table gives the error in the model fit when the parameters from the uni-modal approach are used. Similarly, RMSRE (Classical-) shows the error when the parameters from the classical two-fluid model approach is used. Hence, the improvement in the error from RMSRE (Classical-) to RMSRE (Uni-) can be attributed to the segregation of modes and applying the two-fluid assumptions to each mode. The parameter $\breve{n}$ is highest for cars, followed by taxis, medium vehicles and PTWs. On the other hand, buses have the lowest $\check{n}$ values. The lower $\check{n}$ for buses can be attributed to the presence of dedicated bus lanes, where the impact of traffic from other modes is minimized. At the same time, buses have lower free-flow speed and this is due to lower travelling speeds and less aggressive acceleration-deceleration of public transport vehicles. It will be shown later in Fig. 5 that inaccurate fit also contributes to the lower $\check{n}$ value of the buses. Observing the RMSRE (Uni-) and RMSRE (Classical-) values, it is clear that there is a significant improvement in the mean speed estimation for all modes in the uni-modal approach compared to the classical one. Thus, it can be concluded that segregating the modes results in better mean speed prediction and the assumptions of the classical two-fluid model are valid at the modal level. Now, the estimated $v_{f, r, j}$ and $\check{n}_{j}$ values are used in eq. (13) to built a relation 


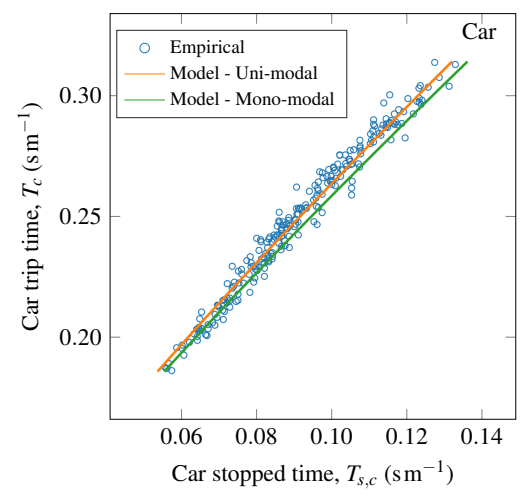

(a)

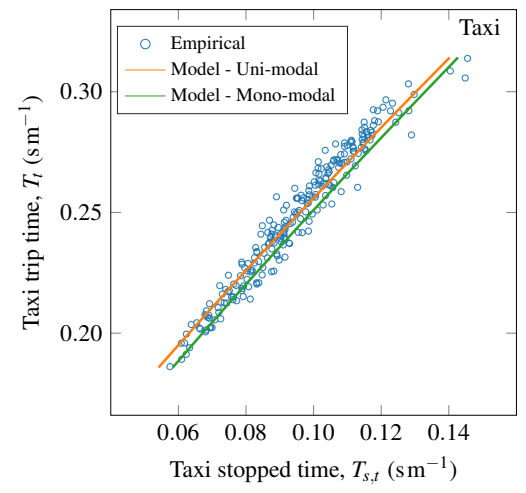

(b)

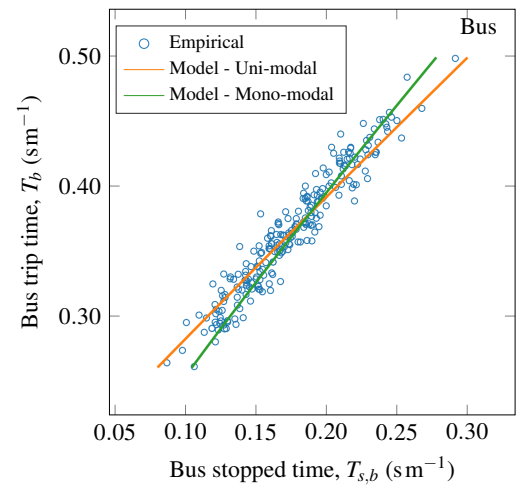

(c)

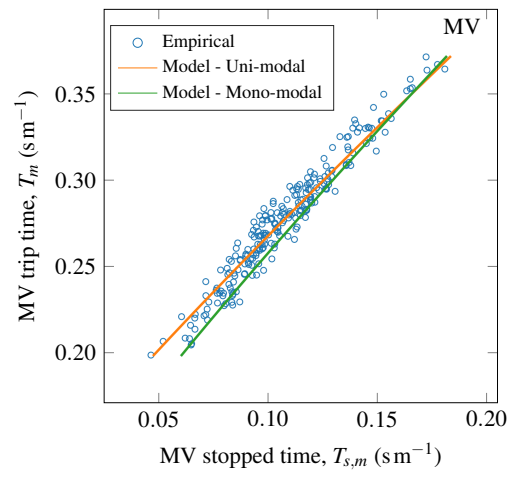

(d)

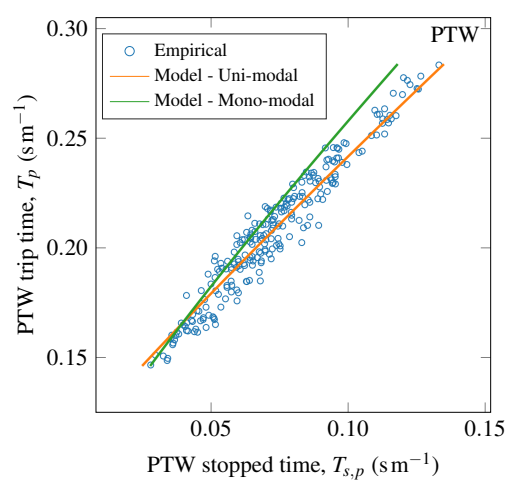

(e)

Figure 5: Comparison of empirical data and model results between stopped time and total time per unit distance in the uni-modal two-fluid regression for all modes considered. (a) Car. (b) Taxi. (c) Bus. (d) Medium Vehicle (MV). (e) PTW.

between trip time $\left(T_{j}\right)$ and stop time $\left(T_{s, j}\right)$ per unit distance. At the same time, both $T_{j}^{\prime}$ and $T_{s, j}^{\prime}$ can be computed empirically from the trajectory data. They can be expressed as follows,

$$
T_{j}^{\prime}=\frac{n_{j}}{P_{j}} \text { and } T_{s, j}^{\prime}=\frac{n_{s, j}}{P_{j}}, \forall j \in\{c, t, b, m, p\},
$$

where $n_{j}$ and $n_{s, j}$ are total and stopped accumulations, respectively and $P_{j}$ is production of mode $j$.

Figure 5 presents empirical data of $T_{j}^{\prime}$ and $T_{s, j}^{\prime}$, estimated by eq. (14), and its corresponding model, using eq. (13) and fit parameters in Table 2. For the sake of comparison, the classical two-fluid model regression is also shown in all plots. Firstly, it is evident that the scatter in the plots is considerably smaller than the ones shown in Fig. 3, where mean speed is expressed as a function of modal accumulations. By considering the trip time as a surrogate to the mean speed, it can be concluded that stopped time (or stopped fraction) is a better variable to characterize mean speed than accumulations per se in the current dataset. At the same time, it can be observed that the empirical data and model are in good agreement for all the modes. Fig. $5 \mathrm{c}$ shows the uni-modal and classical approaches of buses yield skewed results compared to the empirical data. This can be due to several factors like the characteristics of bus, presence of dedicated bus lanes in the bus route, bus bunching, etc., which can increase travel time variability. On the other hand, cars and taxis have a very similar trend as overall traffic behaviour. It can be noticed that the stop times of PTWs are very close to average stop times in the free-flow regime. However, PTWs tend to have fewer stoppages than the network average due to their filtering behaviour in the congestion periods. Fig. 5e shows that the average stop time of PTWs is lower than the network average as the network gets congested.

Thus, it can be concluded that the original two-fluid model can be applied to each mode independently, which in turn helps 
Table 3: Statistical p-values, mean running speed in $\mathrm{ms}^{-1}, R^{2}$ and RMSRE values of uni- and multi-modal two-fluid model fits for all modes using NNLS method.

\begin{tabular}{|c|c|c|c|c|c|c|c|c|c|c|c|c|}
\hline \multirow{3}{*}{ Mode } & \multicolumn{6}{|c|}{$\mathrm{p}$-values } & \multicolumn{2}{|c|}{$v_{f, r, j}$} & \multicolumn{2}{|c|}{$R^{2}$} & \multicolumn{2}{|c|}{ RMSRE } \\
\hline & \multirow{2}{*}{$\begin{array}{l}\text { Uni- } \\
n_{m} \\
\end{array}$} & \multicolumn{5}{|c|}{ Multi- } & \multirow{2}{*}{ Uni- } & \multirow{2}{*}{ Multi- } & \multirow{2}{*}{ Uni- } & \multirow{2}{*}{ Multi- } & \multirow{2}{*}{ Uni- } & \multirow{2}{*}{ Multi- } \\
\hline & & $n_{c}$ & $n_{t}$ & $n_{b}$ & $n_{m}$ & $n_{p}$ & & & & & & \\
\hline Car & 0 & 0 & 0.08 & 0 & 0.25 & 0 & 10.89 & 11.16 & 0.88 & 0.87 & 0.031 & 0.030 \\
\hline Taxi & 0 & 0 & 0 & 0 & 0.89 & 0.01 & 9.81 & 10.45 & 0.79 & 0.85 & 0.035 & 0.030 \\
\hline Bus & 0 & 0 & 0.03 & 0 & 0.33 & 0.08 & 5.78 & 8.21 & 0.66 & 0.77 & 0.076 & 0.063 \\
\hline MV & 0 & 0 & 0.19 & 0 & 0 & 0.61 & 7.89 & 9.35 & 0.77 & 0.83 & 0.046 & 0.040 \\
\hline PTW & 0 & 0 & 0.02 & 0 & 0.67 & 0 & 9.15 & 13.09 & 0.80 & 0.91 & 0.040 & 0.027 \\
\hline
\end{tabular}

to understand the macroscopic behaviour of each mode in the urban network. However, it is clear from the plots that there is still some scatter in the empirical data and the model cannot predict it. The presented uni-modal two-fluid regression is analogous to expressing the mean speed of a given mode as a function of the accumulation of only that mode. By applying the two-fluid model assumptions to each mode independently, the interactions and dependencies between modes are neglected. These multi-modal interactions might be responsible for the scatter in the empirical data.

Figure 6 shows the multi-modal two-fluid parameters for each mode considered in the present work using the NNLS method. Relatively tight fits with high $R^{2}$ and low RMSRE values are obtained for all the modes in the present context. This inference reinforces the previous conclusion that stopped fraction describes the mean speed better than accumulations. Table 3 shows the comparison of $R^{2}$ and RMSRE values between uni- and multi-modal two-fluid approaches. It can be observed that there is a significant improvement in the multi-modal case for PTWs and rather a marginal improvement for the rest of the modes. Furthermore, there is a considerable improvement in the $R^{2}$ values for all modes than the uni-modal case. In cars, the mean speed is mainly influenced by stopped cars and the influence from other modes is negligible as the coefficients are either zero or very low. Hence, the improvement for cars from uni-modal to multi-modal case is minimal. It is clear that stopped buses do not significantly impact the mean speed of cars, but stopped cars impact the mean speed of buses negatively. This can be because passenger cars can use bus lanes to make right turns and tend to make short-stops in the right lanes, where buses normally circulate, negatively impacting their mean speed. Similarly, stopped PTWs have no major influence on the mean speed of cars but stopped cars influence PTWs. This is again due to the filtering phenomenon of PTWs, where the manoeuvring of PTWs between stopped cars reduces the mean speed.

The estimated multi-modal two-fluid regression fit parameters are used to compute stopped time per unit distance using eq. (11). The model estimates are validated using empirical data, as in the previous case. Fig. 7 shows the model estimates and empirical data of stopped time and trip time per unit distance for each mode. Firstly, it can be observed that model estimates yield scatter that is comparable to the empirical data. This phenomenon is entirely absent in the case of the uni-modal two-fluid regression shown in Fig. 5. The presence of scatter in the model is the consequence of assuming the mean speed as a function of the stopped fraction of all modes. This assumption permits to account for the interactions between different modes, which introduce the scatter. Therefore, it can be concluded that the assumptions of the proposed multi-modal two-fluid regression hold and are validated with empirical data. However, it should be noted that the current dataset covers only a minor range of 


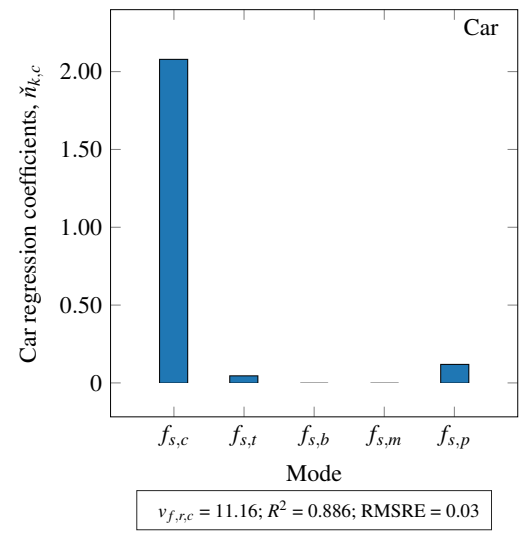

(a)

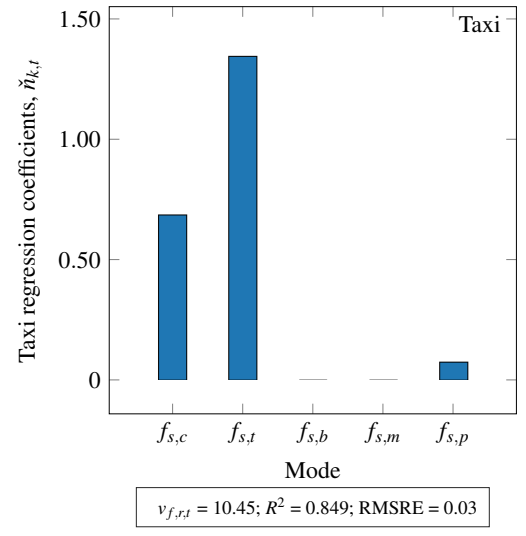

(b)

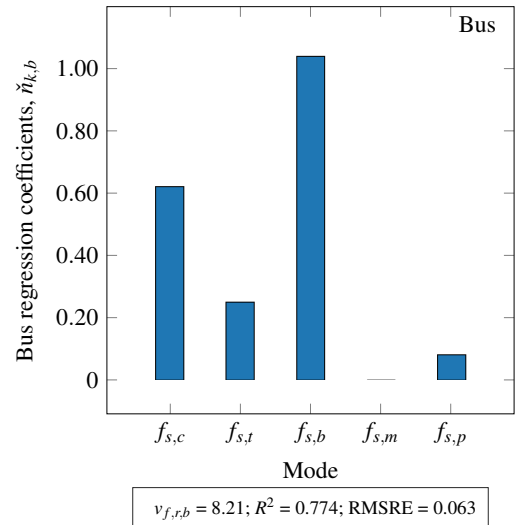

(c)

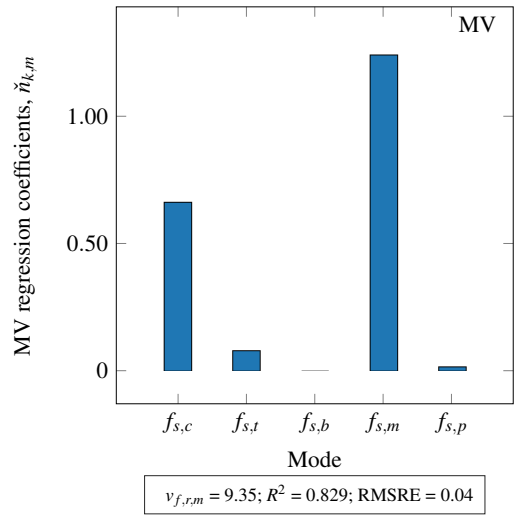

(d)

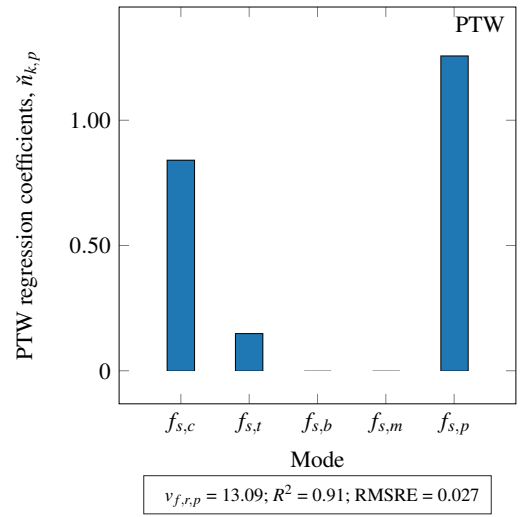

(e)

Figure 6: Multi-modal two-fluid regression fit parameters estimated using NNLS method for each mode. $v_{f, r}$ is in ms ${ }^{-1}$. (a) Car. (b) Taxi. (c) Bus. (d) Medium Vehicle (MV). (e) PTW. 


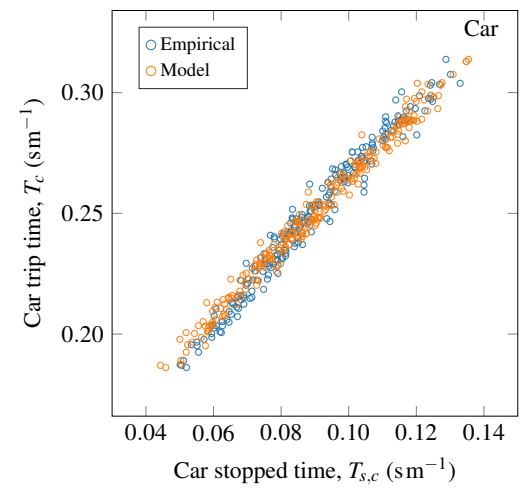

(a)

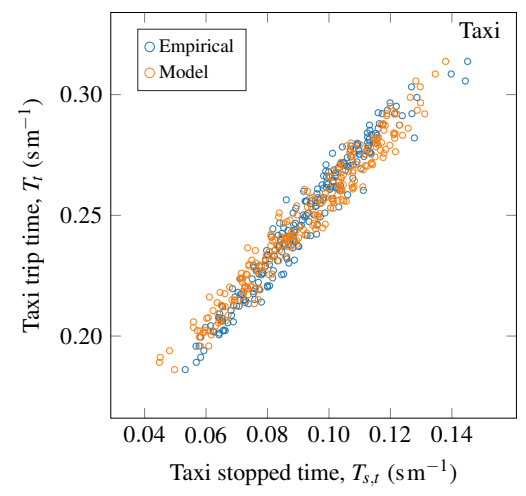

(b)

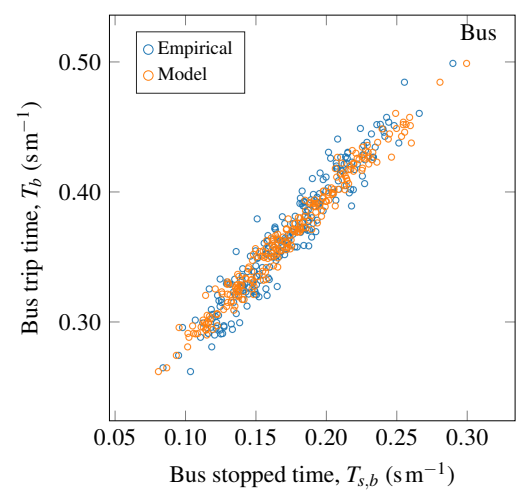

(c)

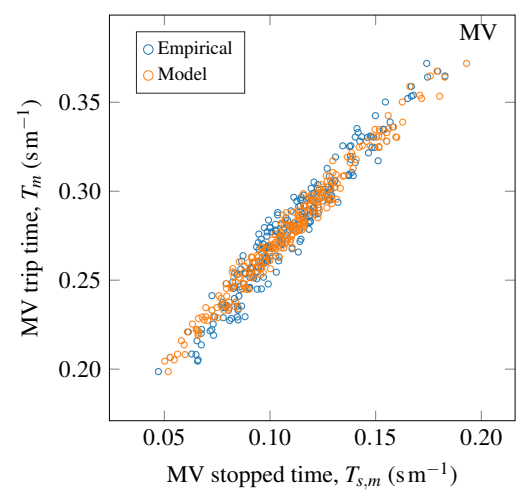

(d)

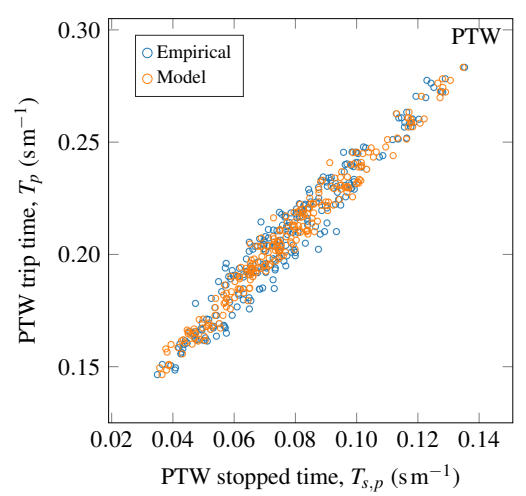

(e)

Figure 7: Comparison of empirical data and model results between stopped time and total time per unit distance in the multi-modal two-fluid regression for all modes considered. (a) Car. (b) Taxi. (c) Bus. (d) Medium Vehicle (MV). (e) PTW. 
mean speeds and it is necessary to validate the model with data that covers a broader range of mean speeds.

Finally, the results from the current model are compared with the ones of multi-modal mean speed analysis as a function of accumulations presented in Section 3. A significant reduction in the RMSRE values for all modes is noticed when the mean speed is expressed in terms of stopped fractions compared to the accumulations. Similarly, there is also improvement in the $R^{2}$ values, which signifies that the stopped fractions can explain the mean speed variability than the accumulations. This improvement is significant for buses and medium vehicles. Therefore, the current work results conclude that the stopped fraction of vehicles better explains the mean speed than the accumulations counterpart. However, it is worth noting that this conclusion is valid only in the current dataset for the network under investigation. More analysis on different networks that cover wider time periods is necessary to arrive at a consensus.

This opens a new research question: how to estimate the stopped fraction of vehicles in the real networks? As in the current case, if GPS traces of individual vehicles are available, the estimation of stopped fractions is trivial. However, the current dataset comes from a unique experiment designed to record traffic observations and these kinds of data are one of their kind. In the absence of GPS data, other means of traffic observations, especially Loop Detector Data (LDD), are not relevant in the current context to determine the stopped and trip times per distance travelled. Thus, it is necessary to resort to surrogate models to estimate an approximate stopped fraction as a function of more common traffic variables like accumulations or densities, which is discussed in Appendix B. The following study discusses the validity of ergodicity assumption and the effect of penetration rates of GPS data on the estimation of stopped fractions.

\subsection{Investigation of Ergodicity assumption and vehicle penetration rates for a multi-modal two-fluid model.}

GPS data can be used to estimate stopped fraction and they are more readily available lately from different types of sources like mobile phones, GPS devices, etc. The main limitation of data from these types of sources is that they do not cover entire traffic and hence, they are available at low to moderate penetration rates. Hence, it is vital to study the influence of penetration rates on the estimation of stopped fractions in the real network. This analysis provides the influence of penetration rates on the estimation of stopped fractions using the present dataset.

In practical systems, it is often not possible to estimate time averages due to various limitations. The ergodic theory was developed to address this shortcoming, proposing that the ensemble average can replace the time average. In the case of traffic, the system is ergodic if the performance of a single test vehicle over a sufficiently long period of time is identical to the mean performance of all the vehicles in the system over the same period (Herman and Ardekani, 1984). The assumption can be considered a strong ergodicity assumption (Chakraborty and Srinivasan, 2016) as it states that the ratio of stopped time to trip time of any probe vehicle is equal to the average fraction of stopped vehicles during the same period and it is valid only in steady-state conditions. This is unlikely to hold in reality due to variability in the driver's behaviour, type and characteristics of probe vehicle, etc. This can be relaxed by assuming that the stopped fraction can be determined by the average stopped and trip distances of all probe vehicles within a given time period. This so-called weak ergodic assumption can be expressed as,

$$
\hat{f}_{s}=\frac{\bar{T}_{s}}{\bar{T}},
$$

where $\bar{T}_{s}$ and $\bar{T}$ are mean stopped time and mean trip time of all probe vehicles in a given time period. Moreover, using the mean travel times represent the population average better than the individual data. Ardekani (1984) studied the strong ergodicity assumption by using aerial photographs and chase-car techniques of urban traffic corridors. It was concluded 
that the average stopped fraction can be approximated as an average of the fraction of stopped times of all probe vehicles.

\begin{abstract}
Mathematically, it can be expressed as, penetration rates. the biases in random sampling.
\end{abstract}

However, very few probe vehicles were used in the study. On the contrary, it is the first time that such a high resolution data is available to provide a rigorous and accurate comparison of the ergodicity conjecture. Thus, the current dataset is used to study the validity of the strong and weak ergodicity assumption using the ground truth presented in eq. (12). This section presents the comparison of ground truth $\left(f_{s}\right)$ with the strong $\left(\tilde{f}_{s}\right)$ and weak $\left(\hat{f}_{s}\right)$ ergodicity assumptions for different levels of

As the current dataset is multi-modal, the stopped fraction of vehicles for each mode is estimated and comparison is made for each mode independently. Different penetration rates $(\rho)$ ranging from 0.05 to 0.95 with a step of 0.05 are considered. For each penetration rate, trajectories are randomly sampled to build a set of probe trajectories. It is to be noted that random sampling is done with all modes combined and thus, a constant penetration rate for each mode is not guaranteed. For each penetration rate, both $\tilde{f}_{s}$ and $\hat{f}_{s}$ are estimated for all aggregation intervals. This process is repeated 1000 times to minimize

Figure 8 shows the evolution of RMSRE of stopped fractions computed using strong and weak ergodicity assumptions for different penetration rates. It is clear from the plots that the error reduces rapidly for weak ergodicity assumption with increasing penetration rate. On the other hand, the error remains almost constant for all modes for the stopped fraction estimated by the strong ergodicity assumption. This error saturation is due to the bias introduced by the strong ergodicity assumption in the computation of stopped fraction. It is noticed that the strong ergodicity assumption under-predicts the stopped fraction consistently for all modes at all ranges of the stopped fraction. This inference is in-line with the field experiments reported in Herman and Ardekani (1984), where $\tilde{f}_{s}$ is slightly lower than ground truth. The error falls between 10-23\% for stopped fraction computed by the strong ergodic condition. The penetration rate needed to estimate stopped fraction using weak ergodicity with an RMSRE error of 0.1 is indicated in the plots. In cars, taxis, and PTWs, around $20 \%$ of penetration is enough to accurately estimate the stopped fraction. However, buses and medium vehicles need more than $50 \%$ penetration rates, which is unrealistic. This can be again due to fewer buses and medium vehicles than other modes in the current data. In the case of buses, this limitation is less critical as bus trajectories can be obtained (even in real-time) from public transit operators and thus, stopped fraction estimates can be extracted accurately. Thus, it can be concluded that the ergodic assumption is valid in the case of the two-fluid model, albeit it should be applied weakly to estimate the stopped fraction accurately. Very good estimates of the stopped fraction can be obtained with lower penetration rates using the weak ergodicity assumption. In the absence of GPS traces, it is not trivial to estimate stopped fractions using classical traffic measurements like LDD.

\section{A Dynamic Trip-based Model Based On Stopped Fraction of Vehicles}

\subsection{Model description}

Dynamic models to predict the evolution of accumulation with time have been developed and integrated in perimeter control strategies based on flow conservation equations (Ramezani et al., 2015). However, it is concluded from the previous analysis that integrating a multi-modal two-fluid model can have a better estimation of the running speed at a given time 


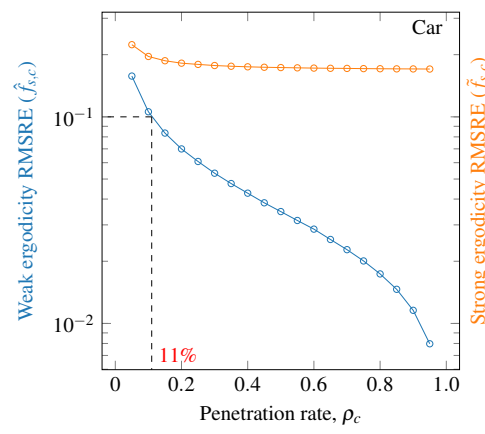

(a)

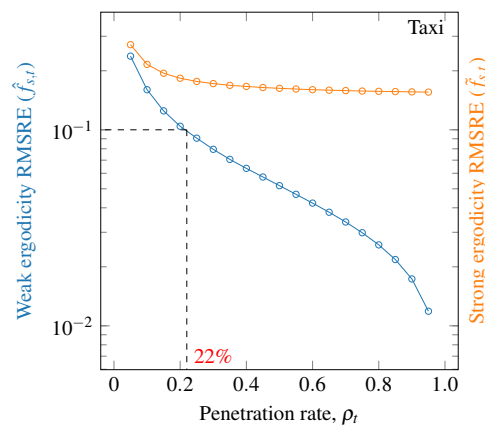

(b)

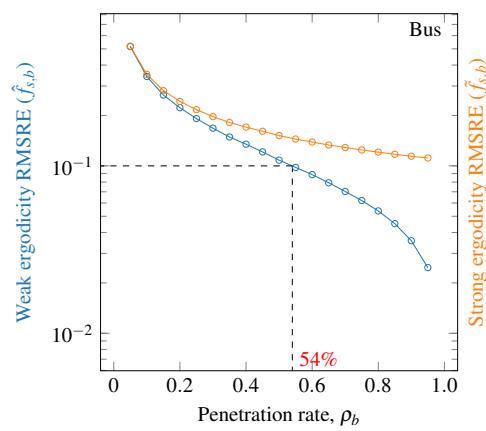

(c)

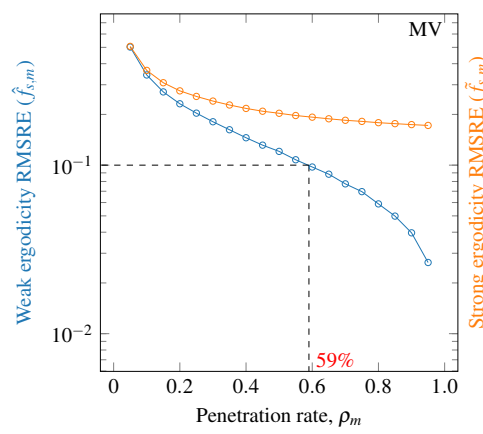

(d)

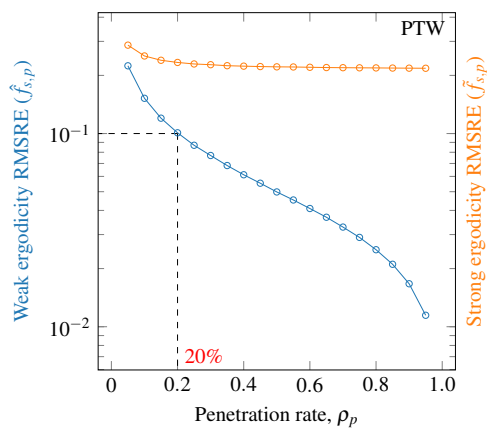

(e)

Figure 8: RMSRE of stopped fractions estimated by the strong $\left(\tilde{f}_{s, j}\right)$ and weak $\left(\hat{f}_{s, j}\right)$ ergodic assumptions with respect to ground truth. The penetration rates indicated in red correspond to an error of 0.1 in $\hat{f}_{s, j}$. (a) Car. (b) Taxi. (c) Bus. (d) Medium Vehicle (MV). (e) PTW.

interval, if the stopped fraction of vehicles for individual modes can be measured. Nevertheless, describing in a dynamic framework the evolution of this fraction is challenging as physical interpretation of such a model is not possible. In this section, a dynamic model that combines concepts both from MFD and two-fluid model is attempted for the first time. In the current MFD-based models, vehicles inside the reservoir always travel with a mean speed given by the MFD until they exit the reservoir. Thus vehicles are not making any stops inside the reservoir in the classical MFD-based approach. Therefore, it is not possible to estimate the stopped fraction of vehicles from the classical MFD-based framework. Simultaneously, the two-fluid model is mainly descriptive about traffic states. It does not possess a dynamic equation to predict traffic evolution by estimating the stopped fraction of the vehicles. It is also shown in Appendix B that there is no well-defined functional form between stopped fraction of vehicles and accumulations. A novel method is proposed in the present work to emulate the stop-and-go patterns of the vehicles on a macroscopic scale. The trip-based MFD approach (Arnott, 2013; Daganzo and Lehe, 2015; Mariotte et al., 2017) is an appropriate choice in this context as trips are already described at an individual level. Mathematically, classical trip-based formulation can be expressed as,

$$
L_{j}=\int_{t-\tau_{j}(t)}^{t} v_{j}\left(\left\{n_{k}(s)\right\}_{k \in \mathscr{M}}\right) \mathrm{d} s \quad \forall j
$$

Consider $\tau_{j}(t)$ is the travel time of a user of mode $j$, who entered the reservoir at time $t$. The mean speed at each time instant depends on the accumulation of each mode in the reservoir, which is given by multi-modal mean speed MFD. Hence, the area under the speed-time curve between the times, $t-\tau_{j}(t)$ and $t$ gives the total travel distance, which is trip length $L_{j}$. By considering an alternation of moving distances with a running speed given by a two-fluid model and stopping intervals for each trip, accumulations of stopped and moving vehicles can be estimated. The distribution of trip length, moving distances 

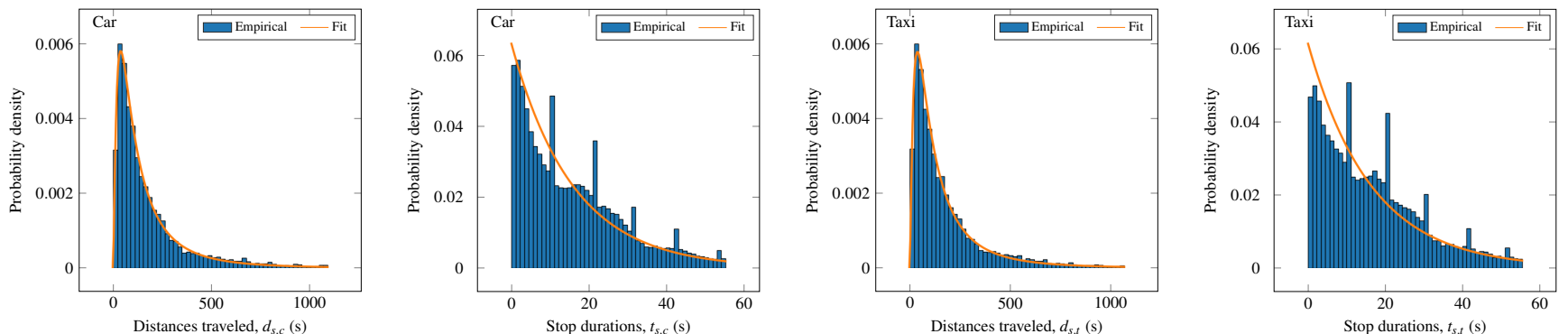

(a)

(b)
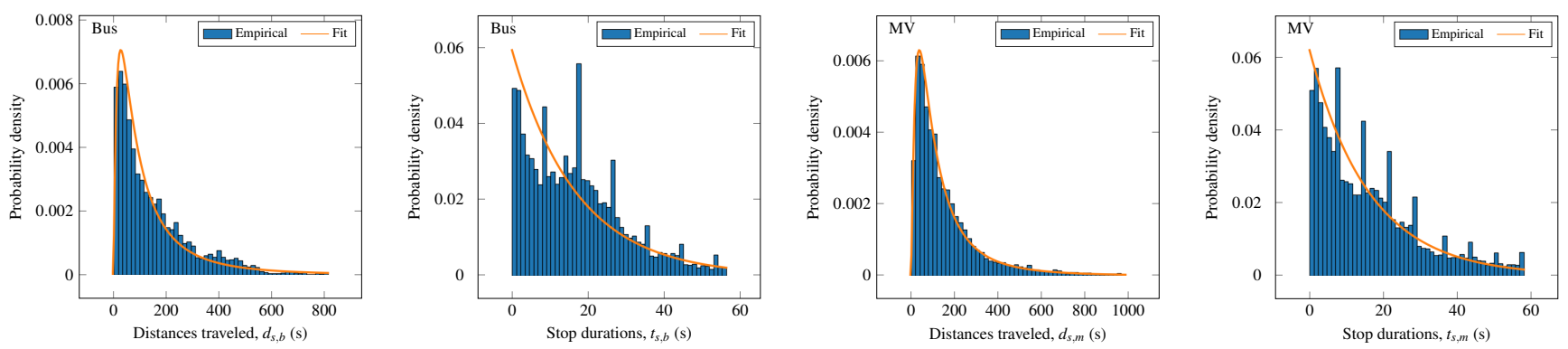

(c)
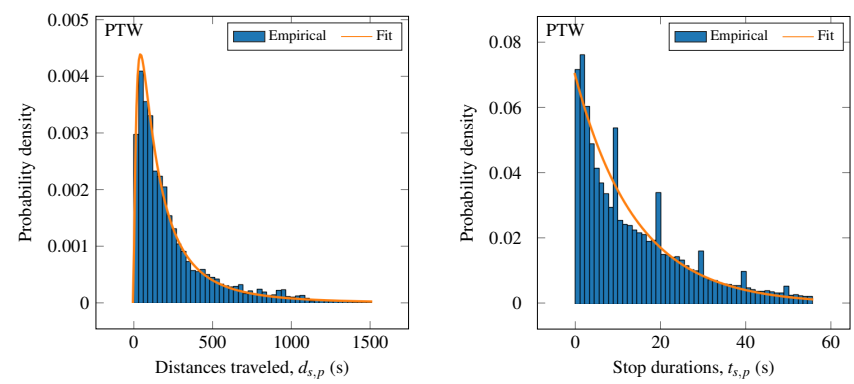

(d)

(e)

Figure 9: Distributions of stop durations and distance travelled between two stoppages. (a) Car. (b) Taxi. (c) Bus. (d) Medium Vehicle (MV). (e) PTW.

and stopping intervals are estimated from the pNEUMA dataset. When stop-and-go patterns are mimicked at the vehicular level, it is trivial to estimate the number of stopped vehicles at any given time and thus, mean speed vs. stopped fractions regression fits can be integrated into the trip-based framework. Consequently, eq. 17 can be re-written as,

$$
L_{j}=\int_{t-\tau_{j}(t)}^{t} v_{j}\left(\left\{f_{k}(s)\right\}_{k \in \mathscr{M}}\right) \mathrm{d} s \quad \forall j,
$$

where the stopped fraction of vehicles, $f_{k}$, is used to estimate the mean speed using multi-modal two-fluid model. Thus, this section discusses the so-called extended trip-based model, which uses mean speed $v s$. stopped fraction as the underlying input to the model and compares the numerical results with the empirical data.

Figure 9 shows the distributions of stop durations $\left(t_{s, j}\right)$ and distances travelled between stoppages $\left(d_{s, j}\right)$ for mode $j$ for all the four days considered. Firstly, it is noticed that the distance between stoppages follows well-defined log-normal distribution for all the modes. Similarly, stop durations follow approximately an exponential distribution in the present case. Exponential and log-normal distributions are fitted to the empirical data of stop durations and distances travelled, respectively. The obtained probability distributions are shown as lines in the plots. It is clear that the estimated fit data and empirical data show very good correspondence for distances travelled.

The distributions presented in Fig. 9 can be considered static as the dynamic information is neglected. The mean stopped 


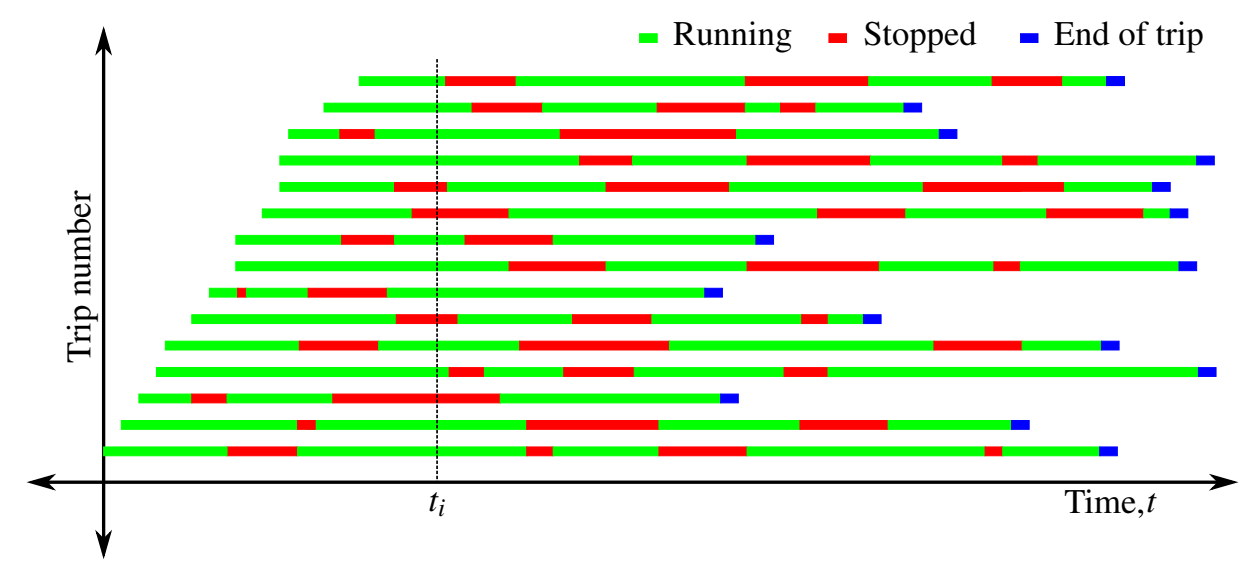

Figure 10: Schematic illustration of the proposed extended trip-based formulation.

time and distance travelled tend to change depending on the traffic conditions. It is noticed that the stop durations are almost

constant at all times. On the other hand, a decrease in the mean distances travelled between stoppages with time is noticed in the current dataset. This observation is in accordance with the mean speed data, where a reduction in mean speed is noticed with time in the data. Recall from Section 2 that each day has five different recording sessions with a duration around 15 min separated by $30 \mathrm{~min}$ breaks. Well-defined distributions of stop durations and distances travelled are obtained inside each session for a given day and hence, these pseudo-dynamic distributions are used in the extended trip-based model.

\subsection{Extended trip-based model}

As stated earlier, typically, vehicles stop and move alternatively in an urban network during their trips. In a simplistic scenario, if the distance travelled between two stoppages and the stop duration are the same for all trips, this so-called stopand-go pattern can be replicated in the trip-based model by allowing the vehicles to travel the given distance before stopping them for the given stop duration alternatively. In reality, these distances and stop durations are rarely constant and can vary widely across the network. However, it is shown in Fig. 9 that reasonably well-defined distributions exist in the current case and hence, it is possible to replicate the stop-and-go pattern of vehicles on a macroscopic scale.

The extended trip-based model requires the following inputs: departure times, trip lengths, mean running speed MFD, distribution of stop durations and distance travelled between two stoppages. Stoppage times and distance covered between stoppages are randomly drawn from the given distributions and assigned to each trip alternatively to reproduce the stop-and-go pattern. Since the stop durations are explicitly accounted for in the simulation with this modified approach, the vehicles must travel with mean running speed between two stoppages and not mean speed. This approach can simulate the stop-and-go pattern of the vehicles at the macroscopic scale. Fig. 10 illustrates the evolution of trips in time in the proposed extended trip-based model. The green zones correspond to the running state and the red ones represent the stoppages. Thus, the stopped fraction of vehicles can be estimated at any given time $t_{i}$ by tracking the number of vehicles that are moving and the vehicles that are stationary at that instant. It is worth noting that these distributions of stop durations and distance travelled are inherently dynamic in nature. In the current context, static distributions inside each session interval are assumed to stop durations and distances travelled. This is equivalent to assigning static distributions for every $15 \mathrm{~min}$, which is a reasonable assumption. Usually, the public transit operators can track the number of buses operating in the network at a given time using Automatic Vehicles Locators (AVL). Consequently, there is no strong necessity to model the accumulation of buses as this 

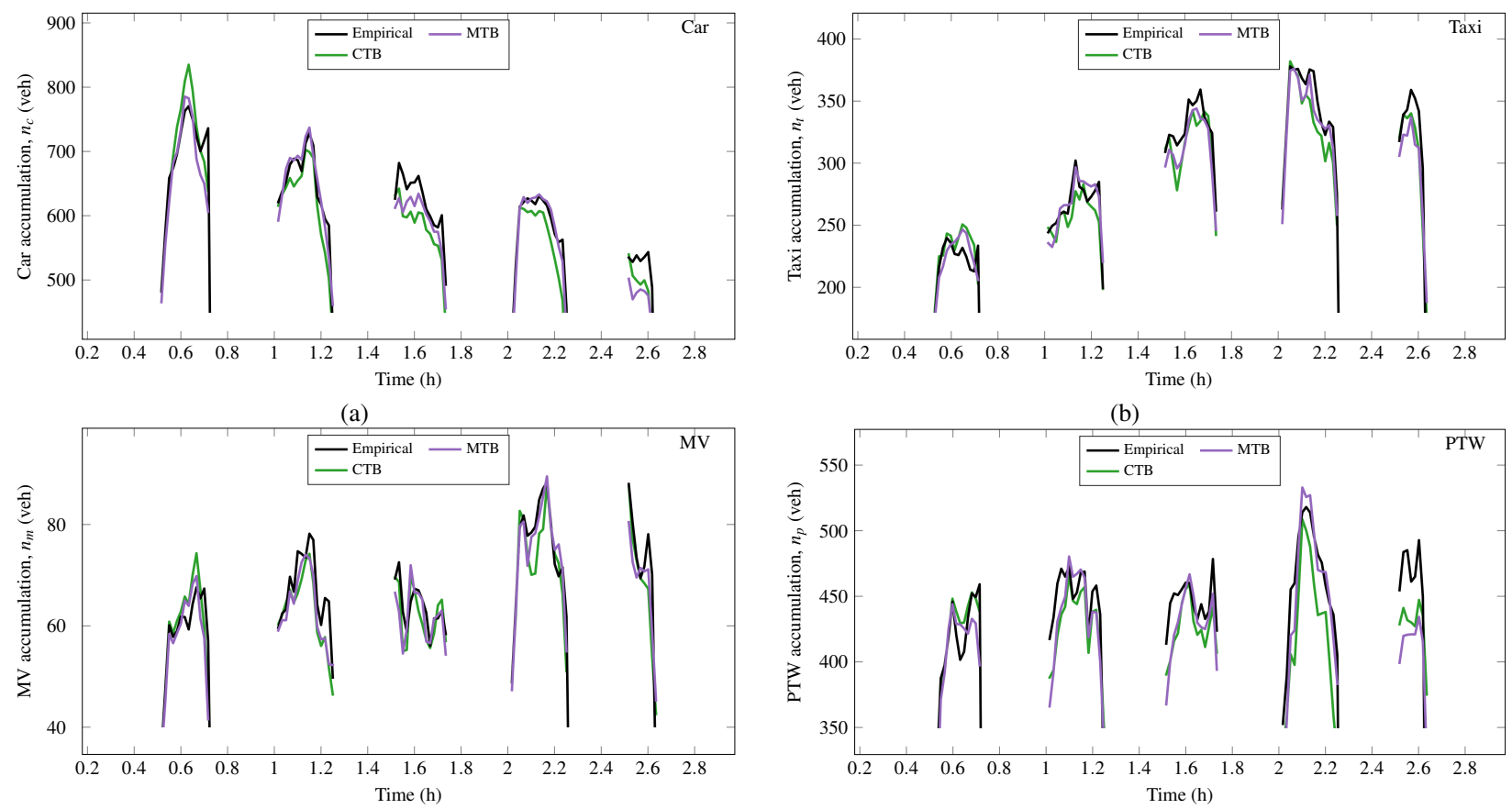

(c)

(d)

Figure 11: Numerical results of Classical Trip-Based (CTB) Extended Trip-Based (ETB) model along with the empirical data for day 1. (a) Car. (b) Taxi. (c) Medium Vehicle (MV). (d) PTW.

information can be obtained from operators in real-time. Hence, in the current work, the accumulation of buses is assumed as known a priori and is provided as an input to the extended trip-based model. For the sake of clarity, only one day is selected to compare the trip-based model results with the empirical data. It is worth noting that mean running speed MFD vs. stopped fraction fits are estimated for each day separately. This minimizes the errors induced by the estimated fits and ensures that the significant differences between numerical and empirical data are due to the modelling framework.

Figure 11 shows the evolution of accumulation for different modes using the proposed extended trip-based model (ETB) for day one along with the empirical data for comparison. Since there are five different sessions, trip-based model is provided with initial accumulations, departure times. Then, the model is run for each session to predict the traffic states based on the mean speed determined by the stopped fraction of vehicles. It can be observed from the empirical data that evolution of accumulation shows sort of loading and unloading behavior. This is due to the synchronization issues during that data collection. At the start and end of the recording periods, not all drones are perfectly synchronized to observe all the data. Consequently, the data observed during the first and last couple of minutes inside each period covers only partial traffic data. Thus, we see this kind of loading and unloading trends in the accumulation. Firstly, it can be observed that the evolution of accumulation in the extended trip-based model is closer to the empirical data for the majority of session intervals. Apart from certain time periods, good accordance is found between empirical data and extended trip-based for most data. It is very difficult to reproduce the exact stopped fraction evolution, even on a macroscopic scale, due to strong constraints on distributions of distances travelled and stop duration. In order to demonstrate the potential gain in the accuracy using the proposed extended trip-based approach, the classical trip-based approach is also considered using multi-modal mean speed MFDs presented in Section 3 and $\mathscr{L}_{2}$ norm of errors are estimated for each day with respect to the empirical data. It is worth noting that mean speed fits are estimated for each day separately and accumulation of buses is used as an input in the classical trip-based model 
Table 4: $\mathscr{L}_{2}$ error norms with respect to the empirical data for Classical Trip-Based (CTB) and Extended Trip-Based (ETB) models.

\begin{tabular}{c|c|c|c|c|c|c|c|c}
\hline \multirow{2}{*}{ Day } & \multicolumn{2}{|c|}{ Car } & \multicolumn{2}{c|}{ Taxi } & \multicolumn{2}{c|}{ MV } & \multicolumn{2}{c}{ PTW } \\
\cline { 2 - 9 } & CTB & ETB & CTB & ETB & CTB & ETB & CTB & ETB \\
\hline 1 & 0.083 & 0.066 & 0.082 & 0.072 & 0.112 & 0.090 & 0.084 & 0.080 \\
2 & 0.116 & 0.086 & 0.113 & 0.086 & 0.164 & 0.111 & 0.137 & 0.104 \\
3 & 0.108 & 0.089 & 0.139 & 0.097 & 0.155 & 0.105 & 0.119 & 0.101 \\
4 & 0.131 & 0.107 & 0.113 & 0.098 & 0.110 & 0.110 & 0.128 & 0.109 \\
\hline
\end{tabular}

515 combining all modes. It was concluded that the uni-modal approach gives a better prediction model compared to the classical 
one with respect to $R^{2}$ and RMSRE values. The uni-modal results were then compared to the multi-modal one, where the mean speed was a function of the stopped fraction of all modes. Again, an improvement was observed in some modes by resorting to a multi-modal approach from uni-modal. Finally, it was noticed that the two-fluid model could better approximate the network-level mean speed than the MFD counterpart in the present work.

Finally, the current analysis was concluded with a discussion on integrating the stopped fractions as variables to the trip-based modelling framework. A novel extended trip-based model was proposed to simulate the stop-and-go pattern of the vehicles. To do so, the distributions of stop duration and distances travelled between the stoppages were introduced in the classical trip-based framework. It was shown that the results of the proposed extended trip-based model were in good accordance with the empirical data. It was also demonstrated by using $\mathscr{L}_{2}$ norm of errors that the extended trip-based model is superior to the classical trip-based.

While MFD research has received significant attention, it is not considered a universal law that can replace all the ambiguities and limitations of microscopic models. Its role is complementary to models and control tools of higher granularity. There are various challenges and ambiguities that require further research efforts. First, the network conditions (in terms of network structure, distribution of congestion and spatio-temporal variations across days) that ensure a well-defined MFD need to be further studied. Second, how data limitations related to measurement errors, spatial coverage and type of sensors influence the observability of network MFDs require further attention. pNEUMA dataset provides a unique opportunity as it contains a level of accuracy in the vehicle positions and penetration rates that have not been observed at just a large scale before. Nevertheless, further experiments of the same type (even for large networks and duration) could shed more light not only in MFD research, but in all granularities of traffic models. Third, how directional congestion influences MFD models and how dynamic clustering can help to better understand network level and scaling effects for multi-modal networks is another challenge. Last but not least, how hierarchical multi-modal traffic management schemes with mixed structure (e.g. upper level perimeter control based on MFD with lower level distributed control) can further alleviate congestion should be further explored.

\section{Acknowledgement}

M. Paipuri and L. Leclercq acknowledge the funding from the European Research Council (ERC) under the European Union's Horizon 2020 research and innovation program (grant agreement No 646592 - MAGnUM project). N. Geroliminis and E. Barmpounakis would like to acknowledge the partial funding supported by the EPFL Open Science Fund and Swiss National Foundation project 200021_188590.

\section{Data Accessibility Statement}

Data source: pNEUMA dataset

\section{Appendix A. Accumulation Coverage and Comparison of Mean Speeds}

Figure A.12 presents the accumulation (first column), mean speed (second column) and mean running speed (third column) of each mode with respect to cars. Firstly, the coverage of accumulations for different modes with respect to cars has a smaller range as the data is only available for limited time periods. The Pearson coefficient between different accumulation pairs is very close to zero, an indication there is no correlation between accumulations. The black line in mean speed and mean 
running speed plots correspond to the line of equal speeds. Firstly, consider mode pair car-taxi in Fig. A.12a. The mean speeds of cars tend to be higher at higher mean speeds (close to the free-flow regime). In contrast, taxis travel faster at lower mean speeds (network saturation/congestion). The lower mean speeds of taxis can be attributed to the service-related stops that taxis make to pick up and drop off passengers or when searching for a new passenger. At the same time, taxis can use the dedicated bus lanes and thus, they can travel at higher speeds than cars, especially during congested periods. It can be inferred that the time gained using the dedicated bus lanes outweighs the time lost in the service-related stops during the peak saturation periods. Hence, the mean speed of taxis is higher than cars at network saturation regimes. Now consider the mean running speeds of the car-taxi mode pair. The mean running speeds of both cars and taxis are almost the same in the entire range of speeds. Again, during the congested periods, taxis are slightly faster than cars, which can be due to the aggressive driving by taxi drivers compared to car drivers. In the case of mode pair car-bus, it is clear that the mean speed and mean running speed of cars are always higher than that of the bus. It is an expected inference as buses tend to travel slower than cars. In the case of medium vehicles, mean speed and mean running speed are always lower than that of cars. The mean running speed of PTWs is higher than that of cars. This is also an expected inference as PTWs tend to be more aggressive and can "filter" through the other urban traffic modes. Finally, it is clear from the plots that the mean speeds of all modes, except buses, tend to coincide with the mean speed of cars at lower speeds (higher congestion) periods. As the network becomes more congested, the vehicles tend to move at the same speed collectively. Buses can be an exception in this scenario as they can travel in the dedicated bus lanes even on the congested road networks. At the same time, the dwell times of the buses lower the overall mean speed and thus, lower mean speeds are obtained even in the congested regimes in the present case.

\section{Appendix B. Relation Between Stopped Fraction And Accumulation}

It is clear from the previous analysis that using stopped fractions of each mode as explanatory variables provides very close fits for the mean speed (or trip time per unit distance). In the absence of GPS data, it is necessary to investigate the relationship between the stopped fraction and other accessible traffic variables like accumulations. Herman and Prigogine (1979) postulated stopped fraction of vehicles can be related to the density $(k)$ as follows,

$$
f_{s}=\left(\frac{k}{k_{c}}\right)^{p}
$$

600

where $k_{c}$ is the maximum density and parameter $p$ measures the quality of traffic. As $p$ increases, $f_{s}$ decreases as $\frac{k}{k_{c}}$ is always less than 1. However, the validity of this relation has not been studied in-detail in the literature. Herman and Ardekani (1984) reported the values of $p$ for Austin and Dallas using average density and stopped fraction values that are obtained from aerial photographs. The present dataset can be used to verify the validity of this assumption. Again, eq. (B.1) was proposed for urban traffic in general, without mode specification. The existence of a relationship in both uni- and multi-modal contexts are investigated. Eq. (B.1) is modified in both approaches as follows,

$$
\begin{aligned}
& \text { Uni-modal: } f_{s, j}=c_{j} n_{j}^{p_{j}}, \forall j \in\{c, t, b, m, p\}, \\
& \text { Multi-modal: } f_{s, j}=c_{j} \prod_{k \in \mathscr{M}} n_{k}^{p_{k, j}}, \forall j \in \mathscr{M} \text { and } \mathscr{M}=\{c, t, b, m, p\},
\end{aligned}
$$

where $c_{j}$ is constant and $n_{k}$ is accumulation of mode $k$. The role of $p_{k, j}$ reverses in the modified relation, i.e., higher $p_{k, j}$ results in higher stopped fractions. Eqs. (B.2) satisfy the boundary conditions, i.e., when $n_{j}=0$, stopped fraction of that mode 

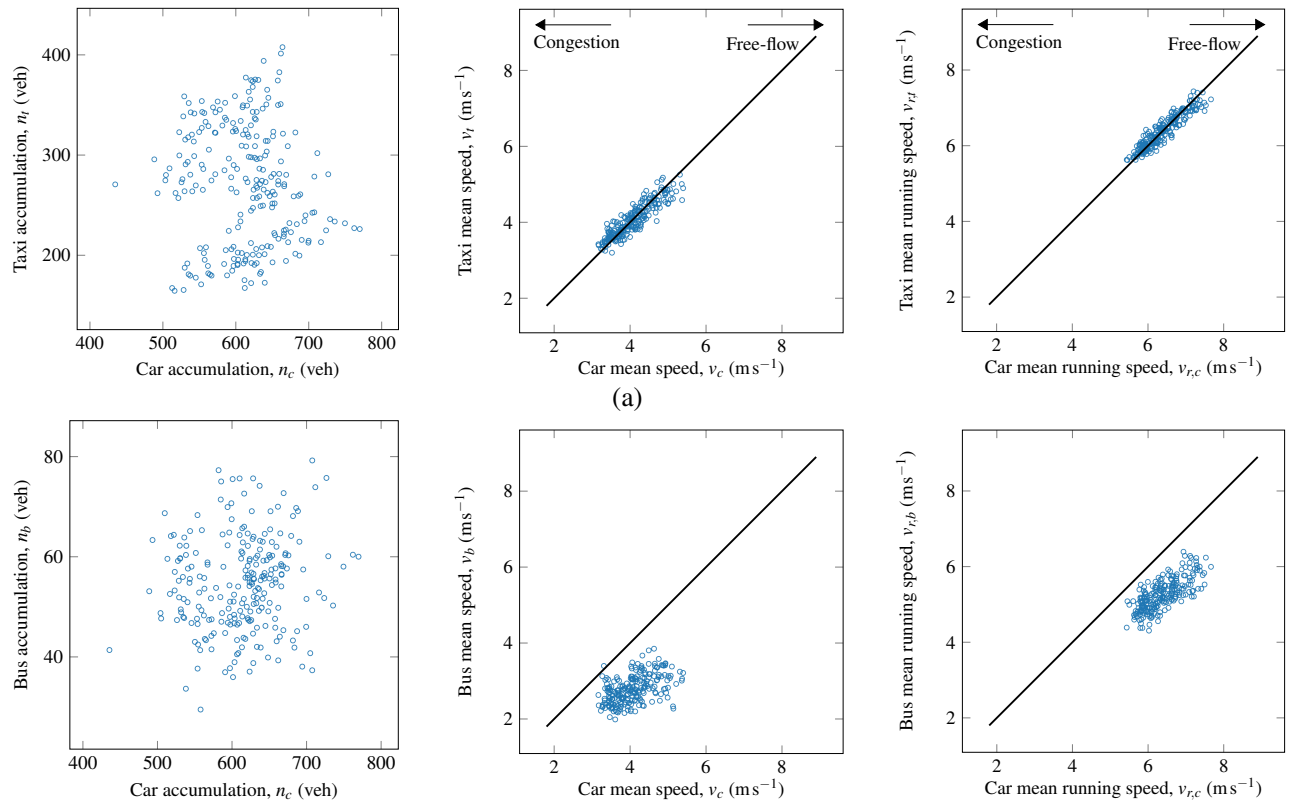

(a)
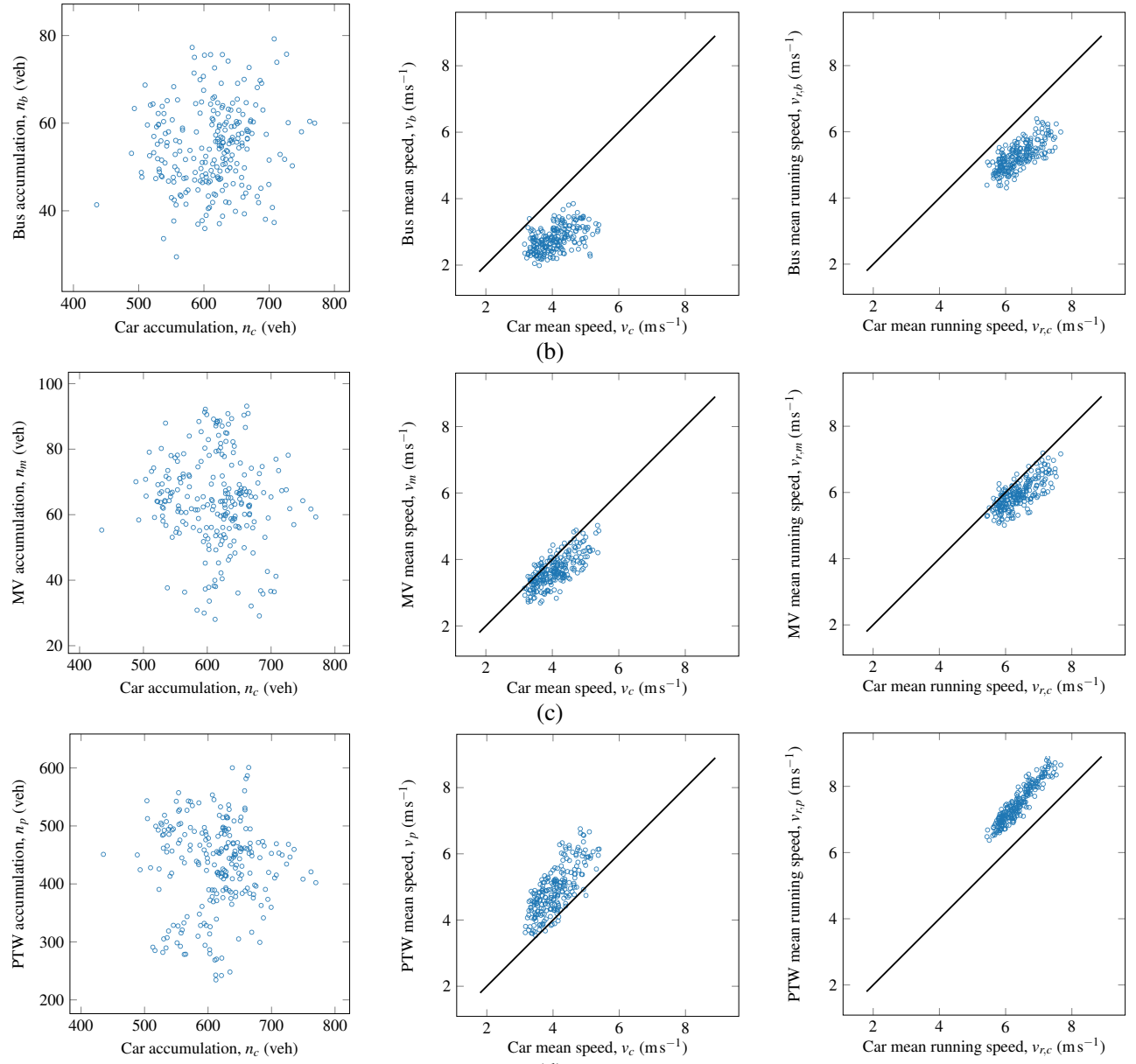

Figure A.12: Comparison of accumulation, mean speed and running speed of each mode with respect to car. (a) Car - Taxi. (b) Car - Bus. (c) Car - Medium Vehicle (MV). (d) Car - PTW. 
Table B.5: Fit parameters for uni-modal relation between stopped fractions and accumulations.

\begin{tabular}{c|c|c|c|c}
\hline Mode & $c_{j}$ & $p_{j}$ & $R^{2}$ & RMSRE \\
\hline Car & 0.363 & 0.014 & 0.02 & 0.093 \\
Taxi & 0.363 & 0.051 & 0.36 & 0.071 \\
Bus & 0.467 & 0.043 & 0.17 & 0.129 \\
MV & 0.383 & 0.048 & 0.18 & 0.108 \\
PTW & 0.342 & 0.123 & 0.54 & 0.106 \\
\hline
\end{tabular}

$f_{s, j}=0$. By using log-transformation, linear regression method NNLS can be used to estimate the regression parameters in eq. (B.2).

Table B.5 presents the fit parameters for the uni-modal case along with the $R^{2}$ and RMSRE values. Relatively poor estimates with larger errors are noticed for most of the modes. It is also worth noting that the values of $p_{j}$ vary across the modes. Figure B.13 shows the regression coefficients of accumulation for predicting stopped fraction using the NNLS technique for different modes. It is clear from the values of $R^{2}$ that accumulations cannot explain the variability in the stopped fractions to a satisfactory level. However, an improvement in both $R^{2}$ and RMSRE values is observed for cars compared to the uni-modal case. The $R^{2}$ values are particularly low for buses and medium vehicles. Consequently, RMSRE values are relatively higher for buses and medium vehicles. This can be due to the scatter in the data or lack of sufficient explanatory variables that can adequately describe the variation in the stopped fraction.

\section{References}

Ambühl, L., Loder, A., Bliemer, M.C.J., Menendez, M., Axhausen, K.W., 2018. Introducing a re-sampling methodology for the estimation of empirical macroscopic fundamental diagrams. Transportation Research Record 2672, 239-248. URL: https://doi.org/10.1177/0361198118788181, doi:10.1177/0361198118788181, arXiv:https://doi.org/10.1177/0361198118788181.

Ampountolas, K., Zheng, N., Geroliminis, N., 2017. Macroscopic modelling and robust control of bi-modal multi-region urban road networks. Transportation Research Part B: Methodological 104, 616 - 637. URL: http://www. sciencedirect. com/science/article/pii/S0191261515300370, doi:https://doi.org/10.1016/j.trb.2017.05.007.

Ardekani, S., Herman, R., 1987. Urban network-wide traffic variables and their relations. Transportation Science 21, 1-16. URL: https://doi.org/10.1287/trsc.21.1.1, doi:10.1287/trsc.21.1.1, arXiv:https://doi.org/10.1287/trsc.21.1.1.

Ardekani, S.A., 1984. The two-fluid characterization of urban traffic : theory, observation, and experiment. Ph.D. thesis. University of Texas. Austin.

Arnott, R., 2013. A bathtub model of downtown traffic congestion. Journal of Urban Economics 76, 110 - 121. URL: http: // WWW.sciencedirect.com/science/article/pii/S0094119013000107, doi:https://doi.org/10.1016/j.jue. 2013.01 .001$. 


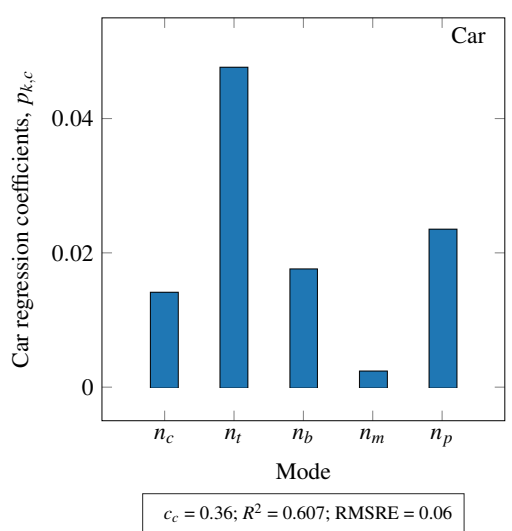

(a)

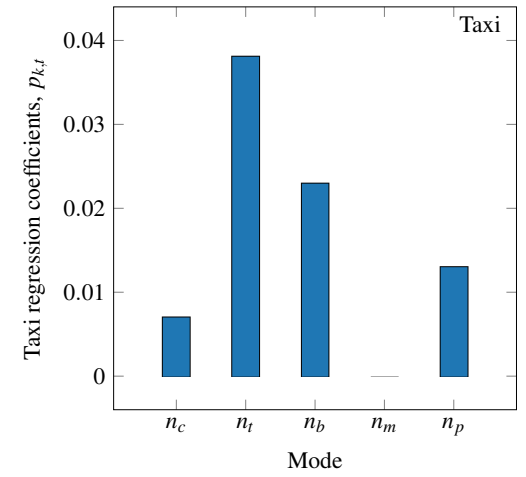

$c_{t}=0.36 ; R^{2}=0.444 ;$ RMSRE $=0.065$

(b)

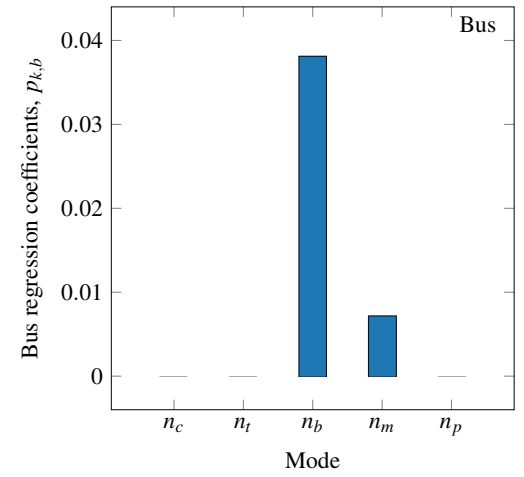

$c_{b}=0.47 ; R^{2}=0.172 ;$ RMSRE $=0.128$

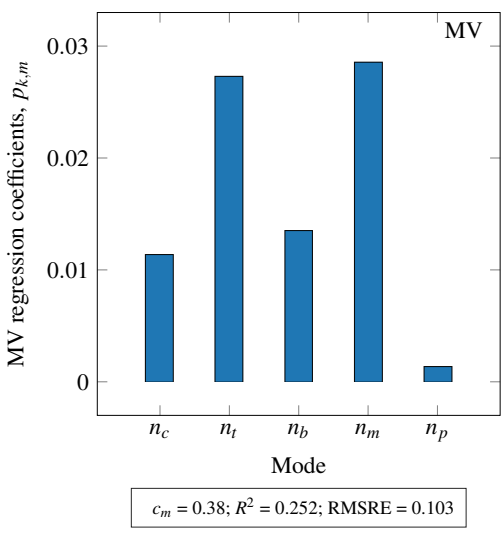

(d)

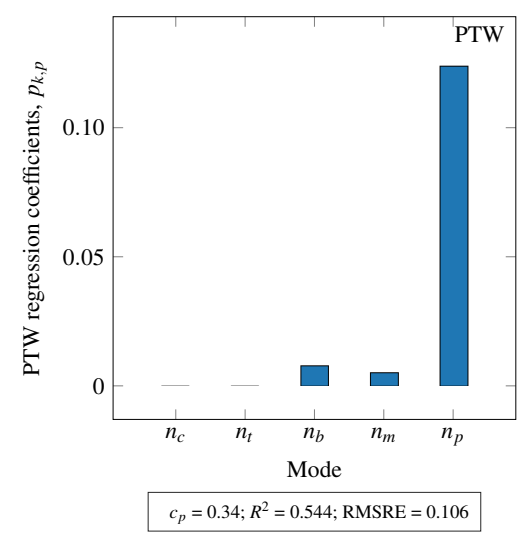

(e)

Figure B.13: Regression coefficients for relation between stopped fraction and accumulation estimated using NNLS method for each mode. (a) Car. (b) Taxi. (c) Bus. (d) Medium Vehicle (MV). (e) PTW. 
Barmpounakis, E.N., Geroliminis, N., 2020. On the new era of urban traffic monitoring with massive drone data: The pNEUMA large-scale field experiment. Transportation Research Part C: Emerging Technologies 111, 50 71. URL: http://www.sciencedirect.com/science/article/pii/S0968090X19310320, doi:https://doi.org/ $10.1016 / j \cdot \operatorname{trc} .2019 \cdot 11.023$.

Barmpounakis, E.N., Vlahogianni, E.I., Golias, J.C., 2016. Intelligent transportation systems and powered two wheelers traffic. IEEE Transactions on Intelligent Transportation Systems 17, 908-916.

Barmpounakis, E.N., Vlahogianni, E.I., Golias, J.C., 2016. Unmanned aerial aircraft systems for transportation engineering: Current practice and future challenges. International Journal of Transportation Science and Technology 5, 111 - 122. URL: http://www.sciencedirect.com/science/article/pii/S2046043016300533, doi:https://doi. org/10.1016/j.ijtst.2017.02.001.

Barmpounakis, E.N., Vlahogianni, E.I., Golias, J.C., 2018. Identifying predictable patterns in the unconventional overtaking decisions of PTW for cooperative ITS. IEEE Transactions on Intelligent Vehicles 3, 102-111.

Barmpounakis, E.N., Vlahogianni, E.I., Golias, J.C., Babinec, A., 2019. How accurate are small drones for measuring microscopic traffic parameters? Transportation Letters 11,332-340. URL: https://doi .org/10 . 1080/19427867. 2017. 1354433, doi:10.1080/19427867.2017.1354433, arXiv:https://doi.org/10.1080/19427867.2017.1354433.

Boyaci, B., Geroliminis, N., 2011. Estimation of the network capacity for multimodal urban systems. Procedia Social and Behavioral Sciences 16, 803 - 813. URL: http://www.sciencedirect.com/science/article/pii/ S1877042811010457, doi:https://doi.org/10.1016/j.sbspro.2011.04.499. 6th International Symposium on Highway Capacity and Quality of Service.

Buisson, C., Ladier, C., 2009. Exploring the impact of homogeneity of traffic measurements on the existence of Macroscopic Fundamental Diagrams. Transportation Research Record: Journal of the Transportation Research Board 2124, 127-136. URL: https://doi .org/10.3141/2124-12, doi:10.3141/2124-12, arXiv:https://doi .org/10.3141/2124-12.

Cao, J., Menendez, M., 2015. System dynamics of urban traffic based on its parking-related-states. Transportation Research Part B: Methodological 81, 718 - 736. URL: http://www.sciencedirect.com/science/article/pii/ S0191261515001654, doi:https://doi.org/10.1016/j.trb.2015.07.018. iSTTT 21 for the year 2015.

Chakraborty, S., Srinivasan, K.K., 2016. Analysis and application of two-fluid model for mixed traffic conditions. Transportation Letters 0, 1-13. URL: https://doi.org/10.1080/19427867.2016.1193309, doi:10.1080/19427867.2016. 1193309, arXiv:https://doi.org/10.1080/19427867.2016.1193309.

Chiabaut, N., Xie, X., Leclercq, L., 2014. Performance analysis for different designs of a multimodal urban arterial. Transportmetrica B: Transport Dynamics 2, 229-245. URL: https://doi.org/10.1080/21680566.2014.939245, doi:10.1080/21680566.2014.939245, arXiv:https://doi.org/10.1080/21680566 . 2014.939245. Methodological 41, 49 - 62. URL: http://www. sciencedirect.com/science/article/pii/S0191261506000282, doi:https://doi.org/10.1016/j.trb.2006.03.001. 
Daganzo, C.F., Lehe, L.J., 2015. Distance-dependent congestion pricing for downtown zones. Transportation Research Part B: Methodological 75, 89 - 99. URL: http://www. sciencedirect.com/science/article/pii/S0191261515000387, doi:https://doi.org/10.1016/j.trb.2015.02.010.

Dixit, V.V., 2013. Behavioural foundations of two-fluid model for urban traffic. Transportation Research Part C: Emerging Technologies 35, 115 - 126. URL: http://www. sciencedirect.com/science/article/pii/S0968090X13001319, doi:https://doi.org/10.1016/j.trc.2013.06.009.

Edie, L.C., 1963. Discussion of traffic stream measurements and definitions, in: The 2nd International Symposium on Theory of Traffic flow, London. pp. 139-154.

Fu, H., Wang, Y., Tang, X., Zheng, N., Geroliminis, N., 2020. Empirical analysis of large-scale multimodal traffic with multisensor data. Transportation Research Part C: Emerging Technologies 118, 102725. URL: http://www. sciencedirect. com/science/article/pii/S0968090X20306409, doi:https://doi.org/10.1016/j.trc.2020.102725.

Gashaw, S., Goatin, P., Härri, J., 2018. Modeling and analysis of mixed flow of cars and powered two wheelers. Transportation Research Part C: Emerging Technologies 89, 148 - 167. URL: http://www.sciencedirect.com/science/article/ pii/S0968090X18301505, doi:https://doi.org/10.1016/j.trc.2018.02.004.

Geladi, P., Kowalski, B.R., 1986. Partial least-squares regression: a tutorial. Analytica Chimica Acta 185, 1 - 17. URL: http://www.sciencedirect.com/science/article/pii/0003267086800289, doi:https://doi.org/10. 1016/0003-2670(86)80028-9.

Geroliminis, N., Daganzo, C.F., 2008. Existence of urban-scale macroscopic fundamental diagrams: Some experimental findings. Transportation Research Part B: Methodological 42, 759 - 770. URL: http://www. sciencedirect.com/ science/article/pii/S0191261508000180, doi:https://doi.org/10.1016/j.trb.2008.02.002.

Geroliminis, N., Zheng, N., Ampountolas, K., 2014. A three-dimensional macroscopic fundamental diagram for mixed bi-modal urban networks. Transportation Research Part C: Emerging Technologies 42, 168 - 181. URL: http:// www.sciencedirect.com/science/article/pii/S0968090X14000709, doi:https://doi.org/10.1016/j.trc. 2014.03 .004$.

Godfrey, J., 1969. The mechanism of a road network. Traffic Engineering and Control 11, 323-327.

Gu, Z., Najmi, A., Saberi, M., Liu, W., Rashidi, T.H., 2020. Macroscopic parking dynamics modeling and optimal real-time pricing considering cruising-for-parking. Transportation Research Part C: Emerging Technologies 118, 102714. URL: https://www.sciencedirect.com/science/article/pii/S0968090X2030629X, doi:https:// doi.org/10.1016/j.trc.2020.102714.

Gu, Z., Shafiei, S., Liu, Z., Saberi, M., 2018. Optimal distance- and time-dependent area-based pricing with the Network Fundamental Diagram. Transportation Research Part C: Emerging Technologies 95, 1 - 28. URL: http:// www.sciencedirect.com/science/article/pii/S0968090X18300573, doi:https://doi.org/10.1016/j.trc. 2018.07.004. 
Haddad, J., Mirkin, B., 2020. Resilient perimeter control of macroscopic fundamental diagram networks under cyberattacks. Transportation Research Part B: Methodological 132, 44-59. URL: https://www.sciencedirect.com/science/ article/pii/S0191261518311457, doi:https://doi.org/10.1016/j.trb.2019.01.020. 23rd International Symposium on Transportation and Traffic Theory (ISTTT 23).

Leclercq, L., Sénécat, A., Mariotte, G., 2017. Dynamic macroscopic simulation of on-street parking search: A trip-based approach. Transportation Research Part B: Methodological 101, 268 - 282. URL: http://www.sciencedirect.com/ science/article/pii/S0191261516309717, doi:https://doi.org/10.1016/j.trb.2017.04.004.

Loder, A., Ambühl, L., Menendez, M., Axhausen, K.W., 2017. Empirics of multi-modal traffic networks-using the 3D www. sciencedirect.com/science/article/pii/S0968090X17301626, doi:https://doi.org/10.1016/j.trc. 2017.06 .009 .

Mahmassani, H., Williams, J., Herman, R., 1984. Investigation of network-level traffic flow relationships: Some simulation results. Transportation Research Record, 121-130. 
Mariotte, G., Leclercq, L., Batista, S., Krug, J., Paipuri, M., 2020. Calibration and validation of multi-reservoir MFD models: A case study in Lyon. Transportation Research Part B: Methodological 136, 62 - 86. URL: http: //www . sciencedirect . com/science/article/pii/S0191261519306769, doi:https://doi.org/10.1016/j.trb.2020.03.006.

Mariotte, G., Leclercq, L., Laval, J.A., 2017. Macroscopic urban dynamics: Analytical and numerical comparisons of existing models. Transportation Research Part B: Methodological 101, 245 - 267. URL: http://www.sciencedirect.com/ science/article/pii/S0191261516307846, doi:https://doi.org/10.1016/j.trb.2017.04.002.

Mohajerpoor, R., Saberi, M., Vu, H.L., Garoni, T.M., Ramezani, M., 2020. $H_{\infty}$ robust perimeter flow control in urban networks with partial information feedback. Transportation Research Part B: Methodological 137, 47 - 73. URL: http:// www.sciencedirect.com/science/article/pii/s0191261518308609, doi:https://doi.org/10.1016/j.trb. 2019.03.010. advances in Network Macroscopic Fundamental Diagram (NMFD) Research.

Paipuri, M., Leclercq, L., 2020. Bi-modal macroscopic traffic dynamics in a single region. Transportation Research Part B: Methodological 133, 257 - 290. URL: http://www.sciencedirect.com/science/article/pii/ S0191261519305612, doi:https://doi.org/10.1016/j.trb.2020.01.007.

Paipuri, M., Xu, Y., González, M.C., Leclercq, L., 2020. Estimating MFDs, trip lengths and path flow distributions in a multi-region setting using mobile phone data. Transportation Research Part C: Emerging Technologies 118, 102709. URL: http://www.sciencedirect.com/science/article/pii/S0968090X20306240, doi:https://doi . org/10.1016/j.trc.2020.102709.

Prigogine, I., Herman, R., 1971. Kinetic Theory of Vehicular Traffic. American Elsevier, New York.

Ramezani, M., Haddad, J., Geroliminis, N., 2015. Dynamics of heterogeneity in urban networks: aggregated traffic modeling and hierarchical control. Transportation Research Part B: Methodological 74, 1 - 19. URL: http:// wWw.sciencedirect.com/science/article/pii/s0191261515000028, doi:https://doi.org/10.1016/j.trb. 2014.12.010.

Saberi, M., Mahmassani, H.S., 2013. Hysteresis and capacity drop phenomena in freeway networks: Empirical characterization and interpretation. Transportation Research Record 2391, 44-55. URL: https://doi .org/10.3141/2391-05, doi:10.3141/2391-05, arXiv:https ://doi.org/10.3141/2391-05.

Saberi, M., Mahmassani, H.S., Hou, T., Zockaie, A., 2014. Estimating network fundamental diagram using three-dimensional vehicle trajectories: Extending edie's definitions of traffic flow variables to networks. Transportation Research Record 2422, 12-20. URL: https://doi.org/10.3141/2422-02, doi:10.3141/2422-02, arXiv:https://doi.org/10.3141/2422-02.

Sirmatel, I.I., Geroliminis, N., 2017. Dynamical modeling and predictive control of bus transport systems: A hybrid systems approach. IFAC-PapersOnLine 50, 7499 - 7504. URL: http://www.sciencedirect.com/science/article/pii/ S2405896317315537, doi:https://doi.org/10.1016/j.ifacol.2017.08.1067. 20th IFAC World Congress.

Sirmatel, I.I., Geroliminis, N., 2018. Economic model predictive control of large-scale urban road networks via perimeter control and regional route guidance. IEEE Transactions on Intelligent Transportation Systems 19, 1112-1121. 
Tsubota, T., Bhaskar, A., Chung, E., 2014. Macroscopic Fundamental Diagram for Brisbane, Australia: Empirical findings on network partitioning and incident detection. Transportation Research Record 2421, 12-21. URL: https://doi.org/ 10.3141/2421-02, doi:10.3141/2421-02, arXiv:https://doi.org/10.3141/2421-02.

Vo, P.T., Mattingly, S.P., Ardekani, S., Dilshad, Y., 2007. Comparison of quality of service in two central business districts: Two-fluid model approach in texas. Transportation Research Record 1999, 180-188. URL: https://doi .org/10.3141/ 1999-19, doi:10.3141/1999-19, arXiv:https://doi.org/10.3141/1999-19.

Wei, B., Saberi, M., Zhang, F., Liu, W., Waller, S.T., 2020. Modeling and managing ridesharing in a multi-modal network with an aggregate traffic representation: A doubly dynamical approach. Transportation Research Part C: Emerging Technologies 117, 102670. URL: https://www.sciencedirect.com/science/article/pii/S0968090X20305854, doi:https: //doi.org/10.1016/j.trc.2020.102670.

Williams, J.C., 2001. Macroscopic flow models, in: Traffic Flow Theory: A State of the Art Report, Committee on Traffic Flow Theory and Characteristics (AHB45). Federal Highway Administration, Washington D. C.. chapter 6, pp. 1-31.

Wold, S., 1992. Nonlinear partial least squares modelling II. Spline inner relation. Chemometrics and Intelligent Laboratory Systems 14, 71 - 84. URL: http://www.sciencedirect.com/science/article/pii/016974399280093J, doi:https://doi.org/10.1016/0169-7439(92)80093-J. proceedings of the 2nd Scandinavian Symposium on Chemometrics.

Yang, M., Pan, Y., Darzi, A., Ghader, S., Xiong, C., Zhang, L., 2020. A data-driven travel mode share estimation framework based on mobile device location data. arXiv:2006.10036.

Yildirimoglu, M., Geroliminis, N., 2014. Approximating dynamic equilibrium conditions with macroscopic fundamental diagrams. Transportation Research Part B: Methodological 70, 186 - 200. URL: http://www. sciencedirect.com/ science/article/pii/S0191261514001568, doi:https://doi.org/10.1016/j.trb.2014.09.002.

Yildirimoglu, M., Ramezani, M., 2020. Demand management with limited cooperation among travellers: A doubly dynamic approach. Transportation Research Part B: Methodological 132, 267-284. URL: https://www. sciencedirect.com/ science/article/pii/S019126151831138X, doi:https://doi.org/10.1016/j.trb.2019.02.012. 23rd International Symposium on Transportation and Traffic Theory (ISTTT 23).

Yildirimoglu, M., Ramezani, M., Geroliminis, N., 2015. Equilibrium analysis and route guidance in large-scale networks with MFD dynamics. Transportation Research Part C: Emerging Technologies 59, $404-420$. URL: http:// www.sciencedirect.com/science/article/pii/S0968090X15001813, doi:https://doi.org/10.1016/j.trc. 2015.05.009. special Issue on International Symposium on Transportation and Traffic Theory.

Zheng, N., Geroliminis, N., 2020. Area-based equitable pricing strategies for multimodal urban networks with heterogeneous users. Transportation Research Part A: Policy and Practice 136, 357-374. URL: https://www. sciencedirect.com/ science/article/pii/S0965856420305644, doi:https://doi.org/10.1016/j.tra.2020.04.009.

Zheng, N., Rérat, G., Geroliminis, N., 2016. Time-dependent area-based pricing for multimodal systems with heterogeneous users in an agent-based environment. Transportation Research Part C: Emerging Technologies 62, 133 
- 148. URL: http://www.sciencedirect.com/science/article/pii/S0968090X15003745, doi:https://doi. org/10.1016/j.trc . 2015.10.015. 